\title{
Chemo-mechanical coupling in saturated porous media: elastic-plastic behaviour of homoionic expansive clays
}

\author{
Benjamin Loret $^{\mathrm{a}, *}$, Tomasz Hueckel ${ }^{\mathrm{b}}$, Alessandro Gajo ${ }^{\mathrm{c}}$ \\ ${ }^{a}$ Laboratoire Sols, Solides, Structures, B.P. $53 X, 38041$ Grenoble Cedex 9, France \\ ${ }^{\mathrm{b}}$ Department of Civil and Environmental Engineering, Duke University, Durham, NC 27708-0287, USA \\ ${ }^{\mathrm{c}}$ Dipartimento di Ingegneria Meccanica e Strutturale, Università di Trento, via Mesiano 77, 38050 Trento, Italy
}

Received 6 January 2001; received in revised form 31 January 2002

\begin{abstract}
Chemically active saturated homoionic clays are considered in a two-phase framework. The solid phase contains the clay particles, absorbed water and salt. The fluid phase, or pore water, contains free water and salt. Water, and possibly salt, can transfer between the two phases. In addition free water may diffuse through the porous medium. A global understanding of the phenomena involved, namely deformation, transfer and diffusion, is proposed. Emphasis is laid on the chemo-mechanical constitutive equations in an elastic-plastic setting. Elastic chemo-mechanical coupling is introduced through a potential, in such a way that the tangent poro-elasticity matrix remains symmetric. Material parameters needed to quantify the coupling are calibrated from specific experiments available in the recent literature. The elasto-plastic behaviour aims at reproducing qualitatively and quantitatively the typical experimental responses observed on almost pure Na-Montmorillonite clays during chemical and mixed chemo-mechanical loadings. Increase of the salinity of pore water at a constant confinement stress leads to a volume decrease, so-called chemical consolidation. Subsequent exposure to a distilled water solution produces swelling: however, the latter is smaller than the chemical consolidation so that the chemical loading cycle results in a net contractancy, the amount of which increases with the confinement. In fact, plastic yielding takes place at low salinities of pore water, and when it stops, chemical preconsolidation is generated.

Natural clays which contain cations of different species are considered in a companion paper, Gajo et al. [Int. J. Solids Struct., this issue], as they require to account for electro-chemo-mechanical couplings. (C) 2002 Published by Elsevier Science Ltd.
\end{abstract}

Keywords: Physico-chemical couplings; Clays; Swelling; Elasto-plasticity

\section{Introduction}

Swelling is an important property of soils being exploited or fought, or engineered in a variety of circumstances, from laying foundations, to setting hydraulic or contamination barriers, to petroleum

\footnotetext{
${ }^{*}$ Corresponding author. Tel.: +33-4-7682-5298; fax: +33-4-7682-7000.

E-mail address: benjamin.loret@hmg.inpg.fr (B. Loret).
} 
drilling. Swelling may cause a non-uniform soil heave, resulting in damage of over-structures, or weakening of bore walls in petroleum drillings. On the other hand, swelling of bentonite is taken advantage of to build barriers to water flow, or contaminant transport in hazardous and nuclear waste disposal technologies.

Swelling of soils is generally understood to be driven by capillary forces and/or by chemical gradients. The former one, termed matric swelling, occurs when soil is unsaturated. The latter one, termed chemical swelling, is characteristic of the so-called expansive clays, rich in mineral smectite, and occurs when there exists a positive difference in concentration of certain species in the soil and in the water in contact with the soil. Typically, these species are salts of metals ( $\mathrm{Na}, \mathrm{K}, \mathrm{Ca}$, etc.).

This paper aims at a mathematical modeling of expansive clays. Saturation of pore space with water is assumed. Swelling of unsaturated and chemically neutral soils is discussed within a three-phase framework similar to the present one in Loret and Khalili (2000). The formulation developed here does not use parameters measured at the microscopic scale, that is at the scale of the clay clusters. It requires only parameters measured at a macroscopic scale through laboratory tests, like oedometer and triaxial tests. Nevertheless, even if the framework to be developed is macroscopic and does not address directly the mechanisms that occur at the molecular scale, it intends to keep track at the larger scale of these mechanisms which are briefly described first.

\subsection{Current understanding of microscopic mechanisms of chemical swelling}

Fundamental to understanding and modeling of chemically driven swelling of expansive clays are their microstructure and organization of their pore space and water. For smectite, this microstructure (see Fig. 1) is dominated by clusters of parallel arranged platelets of clay mineral separated by $10-20 \AA$ interlamellar pores filled with few one-molecule layers of water, called interlayer water, or internal absorbed water. This water has properties, such as density, or viscosity, substantially different from those of free water. Interlayer water does not flow, even when subjected to high hydraulic gradients, and it deforms together with the solid part of clusters. Clusters are enveloped by external adsorbed water layer, up to seven molecular diameter in thickness. For Ca-Montmorillonites, clusters may contain from 5 to 15 platelets and up to 1000 platelets in compacted clays, and they are generally believed to have random orientation of their parallel platelet systems. Ordinarily, clusters are separated by pores with the characteristic size of the order of $1 \mu \mathrm{m}$ and more. Water that resides in such pores is called free water. Indeed, this water can be displaced by ordinary hydraulic gradients.

Swelling of compacted clays develops in two distinct regimes, Norrish (1954), Posner and Quirk (1954) and Sposito and Prost (1982). During swelling at the lowest water contents, called crystalline swelling, interlamellar space absorbs water, until it is filled with three or four molecular water layers. After the interlamellar space has been saturated with this amount of water, water starts to adsorb to the external surfaces of clay particles, producing osmotic swelling. If relatively large particles exist originally in clay, they split after saturation of the interlamellar space with four water layers, opening new external surfaces where an additional adsorption occurs, Saiyouri et al. (2000). However, the model presented here does not pretend to address such details, and we shall gather crystalline and osmotic swelling under the term chemical swelling and make no distinction between adsorbed and absorbed water, both being parts of the solid phase.

From the physico-chemical point of view, crystalline swelling is widely seen as driven by solvation or hydration mechanism of the counterions, and the negatively charged clay surface, Norrish (1954), Delville (1991), Israelachvili (1992) and Sposito et al. (1999): thus it is critically affected by the clay sheets.

The current understanding of the phenomena involved evolves rapidly following developments in experimental techniques and Monte Carlo and molecular dynamics simulations of the interlamellar water structure. Still the issue of the dominant mechanism remains controversial. Counterion solvation is seen as 


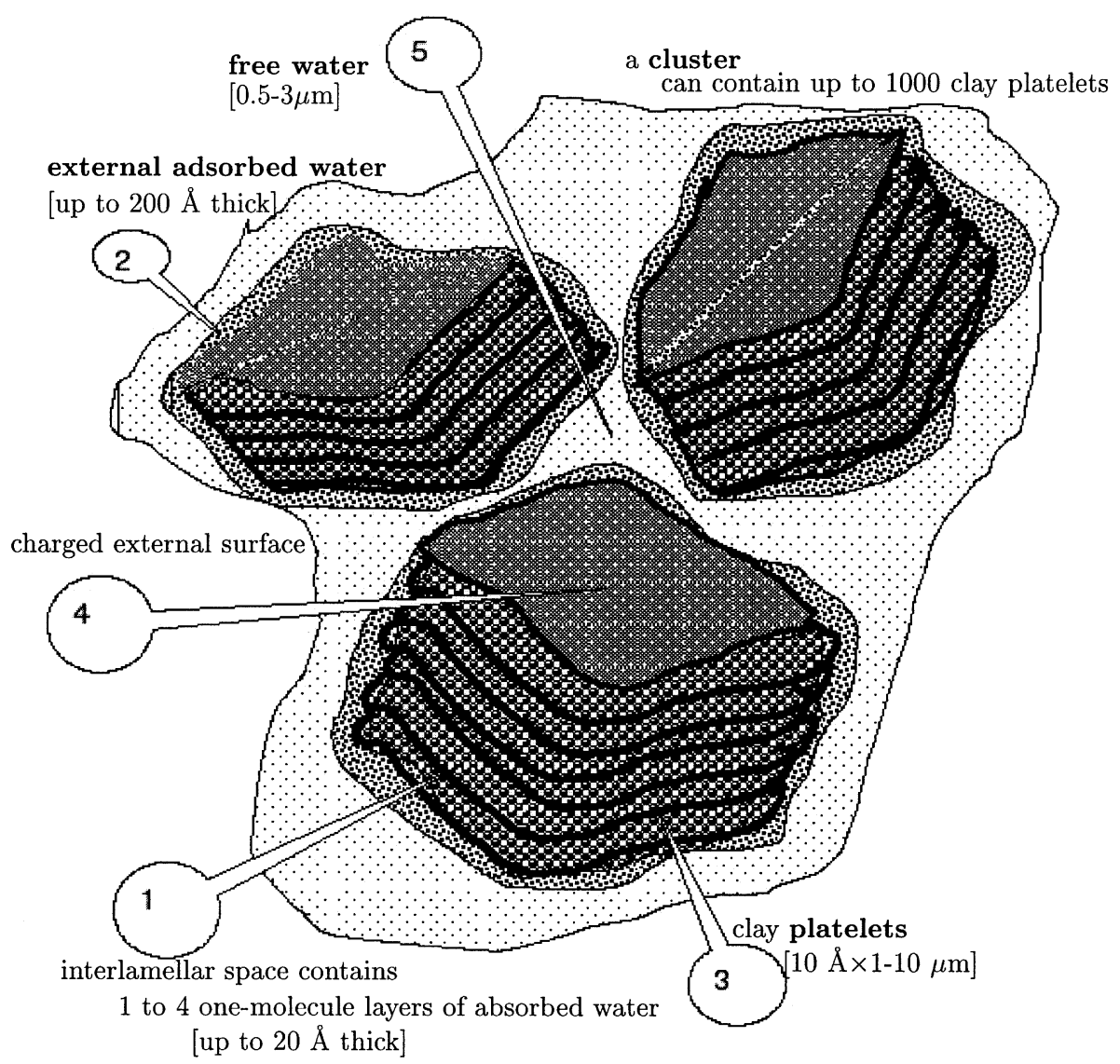

Fig. 1. Cartoon of clay microstructure: (1) interlamellar absorbed water organized in one to four one-molecule thick layers, with watersolvated $\mathrm{Na}^{+}$counterions, gives rise to crystalline swelling; (2) external adsorbed (to the external solid cluster surfaces) water up to 7 molecule diameter thick gives rise to osmotic swelling; (3) silicate solid platelets of clay, about $10 \AA$ thick, negatively charged due to the presence in the surface cavities of isomorphic substitution metals; (4) charged external surface of clay clusters; (5) free water in large $(>1 \mu \mathrm{m})$ intercluster pores.

a dominant mechanism by Sposito and Prost (1982), while hydration of the counterions imbedded in the clay surface is believed to be an equally important cause of swelling by Low (1987), Delville (1991) and others. The solvation mechanism arises due to the presence of the constraining geometry of clay sheet surfaces, and the presence of a cavity in this surface. Most important for our purpose is the finding that solvation forces are proportional to the difference in ionic density between the surface and the mid-plane of the interlamellar space, Israelachvili (1992).

While the distribution of solvating ions and forces can be simulated via equilibrium configuration snapshots, e.g. Karaborni et al. (1996), the mechanisms of changes in the solvation states and transport of water into the interlamellar space are much less understood, Delville (1992). Removal or entrance of liquids from/into nano-slit interlamellar pores appears in Monte Carlo simulations to occur by an instantaneous insertion or depletion of the entire molecular layer, Schoen (1996). There is very little quantitative data, regarding specific nanoscopic mechanisms of the water structuring or transport occurring during the particle spalling and cracking after three to four layer saturation of the interlamellar space, Saiyouri et al. (2000). 
As for swelling due to the adsorption on the existing external surfaces of the particles, it is attributed either to osmotic phenomena, or to a fully developed diffuse electrical double layer.

The mass transfers, that is fluxes, between various types of pores, are postulated to be dependent on the differences in chemical potentials of the individual species, or local ionic concentration midway between the charged platelets, Bolt (1956), van Olphen (1963) and Staverman and Smit (1975). However, pure osmotic analysis leads to a well-known underestimation of the swelling pressure or free swelling deformation, Bolt (1956) and Mitchell (1993). Double layer theory can be suitably calibrated to match experimental findings, Sridharan and Jayadeva (1982), but particulate models of a suspension indicate that the spatial arrangement of particles play a major role in the resulting overall deformation, Anandarajah and $\mathrm{Lu}$ (1991).

As for reversibility of the deformation in swelling, crystalline swelling is largely seen as hysteretic, Boek et al. (1995). Experiments with clay hydration/dehydration indicate a more than $10 \AA$ hysteresis in terms of the layer spacing (12.5\% strain of 1-D parallel layered stack), Fu et al. (1990) and Huang et al. (1994).

However, whether particles tendency to break after absorbing more than four water layers, Saiyouri et al. (2000), is reversible remains unclear. Formation of a system of intercluster cracks in general larger than $200 \AA$ (intrinsic failure) observed by Murray and Quirk (1990), were attributed to hydration-dehydration cycles, suggesting indeed an irreversible formation of separate particles. Accumulation of macroscopic irreversible strain upon cyclic application and removal of $\mathrm{NaCl}$ at large stresses was also observed by $\mathrm{Di}$ Maio (1996).

The above reviewed findings are crucial for the evaluation of the choice of macroscopic or continuum variables to govern fluxes associated with chemical swelling and the significance of the threshold of reversible swelling phenomena.

\subsection{Previous mechanical approaches}

The approach to modeling of the mechanical aspects of clay swelling is typically tailored to the application in hand and specific processes involved.

In petroleum engineering, stability of the wellbore can be improved by circulating a mud that is believed to create a membrane, which is at least partially impermeable to salts: if $p_{\mathrm{m}}, p_{\mathrm{r}}$ are the pressures and $x_{\mathrm{m}}, x_{\mathrm{r}}$ the salt molar fractions of the mud applied to the wellbore and of the rock, respectively, water flow will cease when the chemical potentials equilibrate, which implies the differences $p_{\mathrm{m}}-p_{\mathrm{r}}$ and $x_{\mathrm{m}}-x_{\mathrm{r}}$ to be of the same sign.

For a sufficient difference in salinity between mud and rock, the pore pressure in the rock may at equilibrium be forced to be lower than its initial value, Charlez et al. (1998). Simulations of this phenomenon have been presented by Sherwood (1994a,b), who makes use of a constitutive behaviour referred to as inert, Sherwood (1993): the solid particles constitute the solid phase but the fluid phase contains several species, water and salts, and, at a fixed chemical potential, the mass of a species squeezed out is proportional to its molar fraction. The problem of chemically induced hydration and dehydration of rocks has been addressed by Heidug and Wong (1996): they consider the chemical potentials in equilibrium, i.e. they assume the diffusion processes to occur at a much slower rate than the transfer processes. However, in the above works, transport and transfer phenomena are not addressed separately, that is, it is not recognized that the changes of mass are due to both a transfer (or reactive) contribution and a diffusive contribution. This distinction is necessary to provide elastic constitutive equations that describe coupling effects in swelling clays, and it will be stressed in the present formulation.

Ma and Hueckel (1992) and Hueckel (1992a,b) proposed to treat clays exposed to thermal and chemical loads as a two phase mixture, with absorbed water being a part of solid phase, and an interphase transfer to model its absorption and desorption process. Hueckel (1992a,b) extended Terzaghi's principle of effective 
stress by postulating an a priori equality of the skeletal and absorbed water isotropic partial stresses, and an explicit chemical strain additive with mechanical strain, focusing on its impact on plasticity. Karalis (1993) dealing with simultaneous chemical and capillary swelling discussed a number of ad hoc constitutive functions devised to describe both types of swelling. Bennethum and Cushman (1999) and Murad (1999) have addressed the transfer of water into interlamellar space in clays through a two- or three-spatial scale modeling using homogenization schemes. This type of approach entails a substantial number of constitutive assumptions, requiring sophisticated identification procedures. A multi-constituent mixture, involving charged species and thermal effects, has been suggested by Huyghe and Janssen (1999), but this general framework does not account for the special configuration of clays that will be developed here.

\subsection{A model for chemo-mechanical coupling}

We consider saturated clay as a porous deformable continuum consisting of two overlapping phases, each phase containing several species. However, the strictly physical distinction between the phases may not serve best our purpose, because of ambiguous nature of absorbed water. Adsorbed water has been considered as a third phase by Murad (1999) and endowed with a partial stress, related thermodynamically to the total fluid volume fraction. Here, we will follow a kinematic criterion of phase identification, Hueckel (1992a) and Ma and Hueckel (1992), and attribute the absorbed water to the solid phase based on the affinity of their velocities.

At the heart of the modeling of deformable porous media is the coupling of the deformation of pore space in soil and the concomitant in- or out-flow of pore liquid. In chemically sensitive soils, this coupling is additionally affected by chemical potentials of the species of the solid and fluid phases. By assumption, mechanics of the medium, e.g. balance of momentum, is considered at the phase level, whereas chemical processes, i.e. transport, reaction and balances of masses, concern the species. The link between the two levels is obtained through energetic considerations. Whether or not the chemical potentials are in equilibrium, water absorbed between the clay platelets can transfer into free pore water, or conversely, depending on the chemical composition of the clay and pore water phase, and on the mechanical conditions in terms of volume and pressure. The chemo-mechanical elastic-plastic constitutive equations involve the species present in the solid phase but treat the fluid phase as a whole. The species of the fluid phase only diffuse through the porous medium, obeying generalized diffusion equations. The transfer of absorbed water and species between the solid and fluid phases involves a fictitious membrane surrounding the clay platelets, which may be permeable to the chemical species at various degrees. The transfer equations are time-dependent but, in the present form, they are not intended to imply creep.

The theoretical framework is illustrated by simulations of typical phenomena observed during laboratory experiments: the change of chemical composition of pore water has, due to chemo-mechanical coupling, consequences to the mechanical state of the porous medium. Parameters involved in the model are calibrated. Mechanical, chemical and chemo-mechanical loading and unloading paths are considered. Increase in the salinity of pore water at constant confinement leads to a volume decrease, so-called chemical consolidation. Subsequent exposure to a distilled water solution displays swelling: however, the latter is smaller than the chemical consolidation so that the chemical loading cycle results in a net contractancy whose amounts increases with the confinement. With respect to previous publications, the key feature of the present analysis is that the model developed is not only qualitative but it is able to simulate a rather extensive number of experimental data which have been recently published: these include not only oedometric tests but also triaxial tests in drained and undrained conditions.

Although attention is paid mainly to the elastic-plastic constitutive equations, the latter are shown to be embedded in a general formulation where both transfer and diffusion processes can be considered in initial and boundary value problems to be treated through the finite element method, Gajo and Loret (2001). 
Moreover, clays containing essentially a single counterion, namely Na-Montmorillonite, are considered here: in this situation, the dissolved salt is $\mathrm{NaCl}$ and electroneutrality makes it possible to treat the phases as electrically neutral since chlorine $\mathrm{Cl}^{-}$and sodium $\mathrm{Na}^{+}$transfer together. Counterions are considered to be attached to the negatively charged platelets, and a streaming potential that ensures a vanishing electrical current is limited to a small layer around clay clusters. In a companion paper, Gajo et al. (2002), the framework is extended to include the presence of several counterions, namely sodium $\mathrm{Na}^{+}$and potassium $\mathrm{K}^{+}$, by introduction of electrochemical potentials and enforcement of electroneutrality in both solid and fluid phases.

Notation: Compact or index tensorial notation will be used throughout this note. Tensor quantities are identified by boldface letters. $\mathbf{I}=\left(I_{i j}\right)$ is the second-order identity tensor (Kronecker delta). Symbols '.' and ' $:$ between tensors of various orders denote their inner product with single and double contraction, respectively. tr denotes the trace of a second-order tensor, dev its deviatoric part and div is the divergence operator.

\section{The two-phase framework}

\subsection{General framework}

We consider a two-phase porous medium in isothermal conditions. Each phase is composed of several species:

- the solid phase $\mathrm{S}$ contains three species:

$$
\left\{\begin{array}{l}
\text { clay particles c, } \\
\text { absorbed and adsorbed water w, } \\
\text { salt in absorbed water s, }
\end{array}\right.
$$

- the fluid phase $\mathrm{W}$ contains two species:

$$
\left\{\begin{array}{l}
\text { pore water } \mathrm{w} \\
\text { salt in pore water } \mathrm{s} .
\end{array}\right.
$$

The solid phase presupposes the existence of clusters of particles surrounded by a semi-permeable membrane which is, see Fig. 2, always impermeable to clay particles, and therefore clay suspensions are out of the realm of this study,

$$
m_{\mathrm{cS}}=\text { constant, }
$$

- and either perfect, that is permeable to water only, not to salt; the mass of salt in the solid phase is then constant,

$$
m_{\mathrm{sS}}=\text { constant, }
$$

- or imperfect in the sense that it is permeable to both salt and water at various degrees.

To highlight these aspects, we shall introduce the following sets of species:

- species in solid phase $S=\{w, s, c\}$ and species in fluid phase $W=\{w, s\}$;

- species that can cross the membrane when it is imperfect, $\mathrm{W}^{\leftrightarrow}=\mathrm{S}^{\leftrightarrow}=\{\mathrm{W}, \mathrm{s}\}$;

- species that can cross the membrane when it is perfect, $\mathrm{W}^{\leftrightarrow}=\mathrm{S}^{\leftrightarrow}=\{\mathrm{w}\}$. 


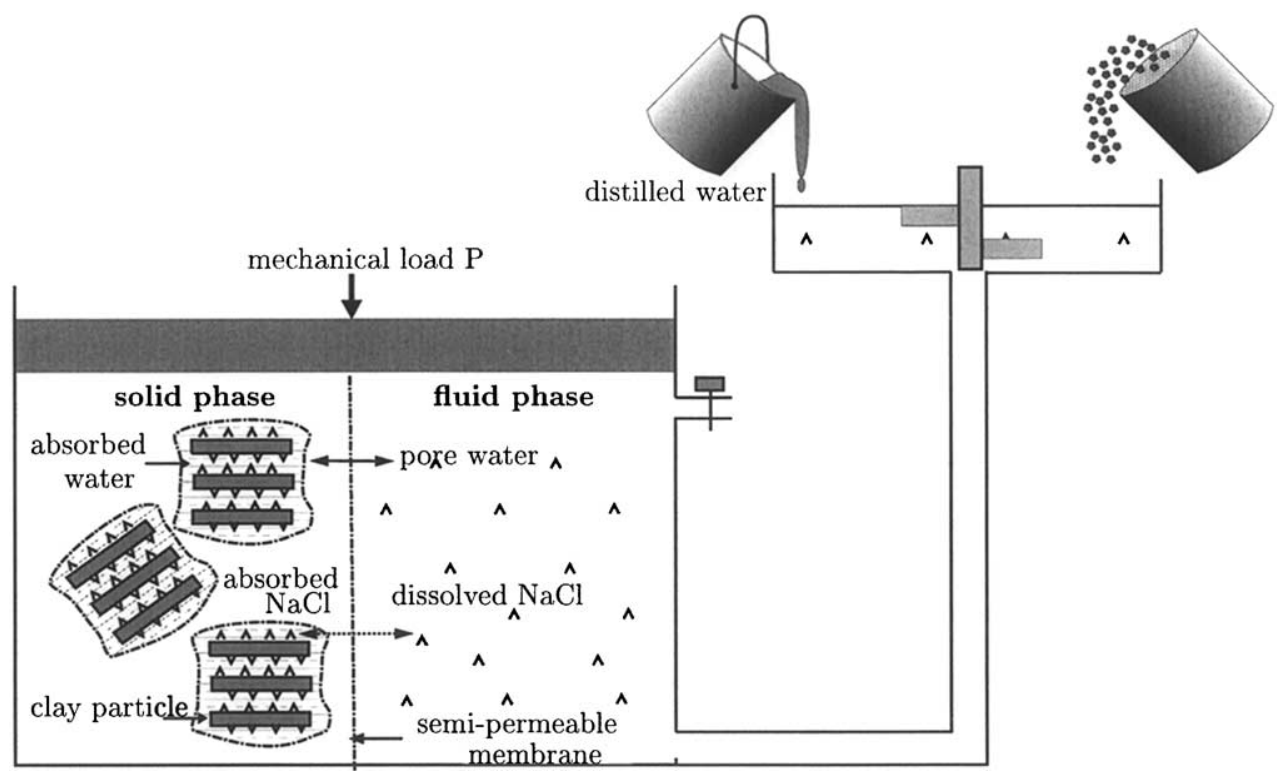

Fig. 2. Schematic of a chemo-mechanical experiment. The pore water is in contact with a large reservoir whose chemical composition is controlled. In the sample submitted to a variable mechanical load in terms of stress or strain, schematized here by $P$, the fluid phase (pore water) and the solid phase (clay clusters with absorbed water and salt) are fictitiously separated by a membrane. Absorbed water is exchanged with pore water depending on the respective salt contents in the two phases and on mechanical conditions. Salt may also transfer between the solid and fluid phases depending on the efficiency of the membrane: due to electroneutrality, a cation $\mathrm{Na}^{+}$and an anion $\mathrm{Cl}^{-}$must transfer simultaneously. The membrane has been delocalized on the sketch, while in fact it surrounds clay clusters.

The main assumptions which underly the two-phase model follow mainly the strongly interacting model of Bataille and Kestin (1977), namely,

(H1) For each species, only the mass balance is required, not the momentum balance.

(H2) Mass balance for each phase is obtained from mass balances of the species it contains. Momentum balances are required for each phase.

(H3) The velocity of each species in the solid phase is that of the phase, $\mathbf{v}_{k \mathrm{~S}}=\mathbf{v}_{\mathrm{S}} \forall k \in \mathrm{S}$, but each species is endowed with its own pressure $p_{k \mathrm{~S}}$. This is motivated by the role that absorbed water may have in transmitting a part of the total stress.

(H4) In the fluid phase, pressure is assumed to be uniform across all species, $p_{k \mathrm{~W}}=p_{\mathrm{W}} \forall k \in \mathrm{W}$, but each species $k$ is a priori endowed with its own velocity $\mathbf{v}_{k \mathrm{~W}}$.

The process of chemical deformation is understood as occuring due to the transfer of mass of water and salt from the pore space into the interlamellar space and/or external surface of clusters and conversely.

\subsection{Basic entities}

In an open system that substances can enter and leave isothermally, the variation of free energy per unit initial volume, i.e. the work done by the total stress $\sigma$ in the incremental strain $\delta \epsilon$ of the solid phase and by the chemical potentials $\mu_{k K}$ during the addition/subtraction of mass $\delta m_{k K}$ of the species $k$ to/from the phase $K$, is 


$$
\delta \Psi=\boldsymbol{\sigma}: \delta \boldsymbol{\epsilon}+\sum_{k, K} \mu_{k K} \delta m_{k K} .
$$

Summation extends to $k=\mathrm{w}, \mathrm{s}$ and $K=\mathrm{W}, \mathrm{S}$ since the contribution due to the clay particles vanishes due to (2.3). The chemical potentials are mass-based ${ }^{1}$ (unit: $\mathrm{J} / \mathrm{kg}$ ) and the $m_{k K}$ 's are the fluid-mass contents per unit initial volume of the porous medium (unit: $\mathrm{kg} / \mathrm{m}^{3}$ ). Since the volume of the solid phase is also the volume of the porous medium, $\operatorname{tr} \epsilon$ is the relative volume change of both these quantities.

The chemical potential of the species $k$ in the fluid phase $\mathrm{W}$ is expressed through the classic formula, e.g. Haase (1990, Chapter 2-5), Kestin (1968, Chapter 21), which includes a purely mechanical contribution involving

- the intrinsic pressure of the fluid phase $p_{\mathrm{W}}$,

- the intrinsic density of the species $\rho_{k \mathrm{~W}}$,

and a chemical contribution which accounts for the molar fraction $x_{k \mathrm{~W}}$ of the species $k$ in phase W. The incremental change of chemical potential reads,

$$
\delta \mu_{k \mathrm{~W}}=\frac{\delta p_{\mathrm{W}}}{\rho_{k \mathrm{~W}}}+\frac{R T}{m_{k}^{(\mathrm{M})}} \delta \ln x_{k \mathrm{~W}}, \quad k=\mathrm{w}, \mathrm{s} .
$$

As for the species within the solid phase $\mathrm{S}$, the mechanical contribution to the chemical potential of species $k$ involves its intrinsic mean-stress $p_{k \mathrm{~S}}$,

$$
\delta \mu_{k \mathrm{~S}}=\frac{\delta p_{k \mathrm{~S}}}{\rho_{k \mathrm{~S}}}+\frac{R T}{m_{k}^{(\mathrm{M})}} \delta \ln x_{k \mathrm{~S}}, \quad k=\mathrm{w}, \mathrm{s} .
$$

In these formulas, $R=8.31451 \mathrm{~J} / \mathrm{mol} \mathrm{K}$ is the universal gas constant, $T(\mathrm{~K})$ the absolute temperature and $m_{k}^{(\mathrm{M})}$ is the molar mass of the species $k$, e.g. $m_{\mathrm{w}}^{(\mathrm{M})}=18 \mathrm{~g}$.

As mentioned earlier, swelling and chemical consolidation involve both interlamellar, (parallel) and (not necessarily parallel) external cluster surfaces, including the conversion of internal surfaces into new external surfaces in particle splitting. The exact mechanisms of interaction of non-parallel surfaces are not known, including the role of intercluster porosity. Since for the materials of the densities of our interest, the dominant number of platelet surfaces are parallel and swelling (or water mass transfer) is controlled by osmotic pressure, we shall refer to that concept in our formulation of chemo-elastic portion of the potential.

The density of absorbed water has been reported to be non-uniform between the platelets and to be slightly higher than that of free water. However, some algebraic simplifications arise if we assume the density of any species to be one and the same in both solid and fluid phases,

$$
\rho_{k \mathrm{~S}}=\rho_{k \mathrm{~W}}, \quad k \in \mathrm{S}^{\leftrightarrow}=\mathrm{W}^{\leftrightarrow} .
$$

One of the principal tasks in building a theory of deformable porous media is to link the change of pore space to the mass of pore liquid flowing in or out of the representative volume element. This description is more complex when reactions take place, resulting in generation or disappearance of mass. We shall define below different measures of mass changes and volume changes that will be used in the constitutive equations.

The molar fraction $x_{k K}$ of the species $k$ in phase $K$ is defined by the ratio of the mole number $N_{k K}$ of that species within the phase $K$, namely

\footnotetext{
${ }^{1}$ Another convention is to use mole-based chemical potentials and measure the amount of substances in moles.
} 


$$
x_{k K}=\frac{N_{k K}}{\sum_{l \in K} N_{l K}} .
$$

Summation extends to $l=\mathrm{w}$, s for $K=\mathrm{W}$, and to $l=\mathrm{w}, \mathrm{s}, \mathrm{c}$ for $K=\mathrm{S}$. The molar fractions satisfy the constraints,

$$
x_{\mathrm{wW}}+x_{\mathrm{sW}}=1, \quad x_{\mathrm{wS}}+x_{\mathrm{sS}}+x_{\mathrm{cS}}=1 .
$$

Let the initial volume of the porous medium be $V_{0}$ and let $V=V(t)$ be its current volume. The current volume of the species $k$ of phase $K$ is denoted by $V_{k K}$ and the current volume of phase $K$ by $V_{K}$. Then the volume fraction of the species $k$ of phase $K$ is defined as

$$
n_{k K}=\frac{V_{k K}}{V},
$$

while the volume fraction of phase $K$ is

$$
n_{K}=\frac{V_{K}}{V}=\sum_{k \in K} n_{k K} \quad \text { with } n_{\mathrm{S}}+n_{\mathrm{W}}=1 .
$$

The ratios of the current volumes to the initial total volume $V_{0}$ introduce the volume contents $v_{k K}$ for the species $k$ of phase $K$ and $v_{K}$ for the phase $K$, namely,

$$
v_{k K}=\frac{V_{k K}}{V_{0}}=n_{k K} \frac{V}{V_{0}}, \quad v_{K}=\frac{V_{K}}{V_{0}}=n_{K} \frac{V}{V_{0}} .
$$

The mass contents $m_{k K}$ per unit initial volume $V_{0}$ of the species $k$ of phase $K$, and $m_{K}$ for the phase $K$, are obtained from the above quantities:

$$
m_{k K}=\frac{M_{k K}}{V_{0}}=\rho_{k K} v_{k K} \quad(\text { no summation on } k, K), \quad m_{K}=\frac{M_{K}}{V_{0}}=\sum_{k \in K} m_{k K} .
$$

The apparent density of the species $k$ in the porous medium, namely $\rho^{k K}$, and the apparent density of the phase $K$, namely $\rho^{K}$, refer to the volume of the porous medium $V$,

$$
\rho^{k K}=n_{k K} \rho_{k K} \quad(\text { no summation on } k, K), \quad \rho^{K}=\sum_{k \in K} \rho^{k K} .
$$

Since $N_{k K}=m_{k K} V_{0} / m_{k}^{(\mathrm{M})}$, the molar fractions $x_{k K}$ can be expressed in terms of the mass contents

$$
x_{k K}=\frac{m_{k K} / m_{k}^{(\mathrm{M})}}{\sum_{l \in K} m_{l K} / m_{l}^{(\mathrm{M})}} .
$$

For the record, let us note the partial derivative,

$$
\frac{\partial x_{k K}}{\partial m_{l K}}=\frac{x_{l K}}{m_{l K}}\left(I_{k l}-x_{k K}\right) \quad \forall k, l \in K .
$$

Let us denote $v_{k K}^{(\mathrm{M})}$ the molar volume of the species $k$ of phase $K$,

$$
m_{k}^{(\mathrm{M})}=\rho_{k K} v_{k K}^{(\mathrm{M})} \quad(\text { no summation on } k, K) .
$$

Note that, if the densities are assumed to be independent of the phases, Eq. (2.8), so do the molar volumes. The molar volume of the fluid phase is

$$
v_{\mathrm{W}}^{(\mathrm{M})}=x_{\mathrm{wW}} v_{\mathrm{wW}}^{(\mathrm{M})}+x_{\mathrm{sW}} v_{\mathrm{sW}}^{(\mathrm{M})} \text {. }
$$

We will also need the densities referred to the fluid phase rather than to the porous medium, usually referred to as mass concentrations, namely 


$$
c_{k \mathrm{~W}}=\frac{\text { mass of species } k \text { of fluid phase }}{\text { volume of fluid phase }}=\frac{M_{k \mathrm{~W}}}{V_{\mathrm{W}}}=\frac{\rho^{k \mathrm{~W}}}{n_{\mathrm{W}}}, \quad k=\mathrm{w}, \mathrm{s} .
$$

From (2.19) and (2.20) follows

$$
c_{k \mathrm{~W}}=\frac{m_{k}^{(\mathrm{M})} N_{k \mathrm{~W}}}{v_{\mathrm{W}}^{(\mathrm{M})}\left(N_{\mathrm{wW}}+N_{\mathrm{sW}}\right)}=\frac{m_{k}^{(\mathrm{M})}}{v_{\mathrm{W}}^{(\mathrm{M})}} x_{k \mathrm{~W}}, \quad k=\mathrm{w}, \mathrm{s} \quad(\text { no summation on } k) .
$$

\subsection{The Gibbs-Duhem relation}

For a fluid phase, the Gibbs-Duhem relation provides the fluid pressure $p_{\mathrm{W}}$ in terms of the chemical potentials of the species $\mu_{k \mathrm{~W}}$, namely Haase (1990, Chapter 1-13),

$$
\delta p_{\mathrm{W}}=c_{\mathrm{wW}} \delta \mu_{\mathrm{wW}}+c_{\mathrm{sW}} \delta \mu_{\mathrm{sW}} .
$$

This relation is easy to retrieve from the definitions above by forming a linear combination of the chemical potentials (2.6) that eliminates the chemical contribution via $(2.10)_{1}$; the result follows by using successively (2.18), (2.19) and (2.21). In order to define the mechanical constitutive equations of the porous medium in Section 4, we will need to isolate the chemical effects in pore water. For that purpose, following Heidug and Wong (1996), we define the chemical energy per current unit volume of the fluid phase through its differential

$$
\delta \psi_{\mathrm{W}}=\mu_{\mathrm{wW}} \delta c_{\mathrm{wW}}+\mu_{\mathrm{sW}} \delta c_{\mathrm{sW}}
$$

which, via the Gibbs-Duhem relation (2.22), can be integrated, up to a constant, to

$$
\psi_{\mathrm{W}}=\mu_{\mathrm{wW}} c_{\mathrm{wW}}+\mu_{\mathrm{sW}} c_{\mathrm{sW}}-p_{\mathrm{W}} .
$$

Using (2.13), (2.14) 1 and (2.20), one has $c_{k \mathrm{~W}} v_{\mathrm{W}}=m_{k \mathrm{~W}}, k=\mathrm{w}$, s. Therefore, the chemical energy of the fluid phase per unit initial volume of porous medium is, using (2.13),

$$
\Psi_{\mathrm{W}}=\psi_{\mathrm{w}} \frac{V_{\mathrm{W}}}{V_{0}}=\psi_{\mathrm{W}} v_{\mathrm{W}}=\mu_{\mathrm{wW}} m_{\mathrm{wW}}+\mu_{\mathrm{sW}} m_{\mathrm{sW}}-p_{\mathrm{W}} v_{\mathrm{W}} .
$$

Indeed, due to the Gibbs-Duhem relation (2.22), the differential of $\Psi_{\mathrm{W}}$ simplifies to

$$
\delta \Psi_{\mathrm{W}}=\sum_{k \in \mathrm{W}} \mu_{k \mathrm{~W}} \delta m_{k \mathrm{~W}}-p_{\mathrm{W}} \delta v_{\mathrm{W}}
$$

\subsection{Incompressibility constraint}

A situation of particular interest arises when all species are incompressible, $\delta \rho_{k K}=0 \forall k, K$. Then, there exists a relation between the set of variables $\left\{\boldsymbol{\epsilon}, v_{\mathrm{W}}, m_{\mathrm{wS}}, m_{\mathrm{sS}}\right\}$. With (2.14), the increment of fluid volume content can be expressed as, Appendix A, Eq. (A.8),

$$
\delta v_{\mathrm{W}}=\delta \operatorname{tr} \epsilon-\frac{\delta m_{\mathrm{wS}}}{\rho_{\mathrm{wS}}}-\frac{\delta m_{\mathrm{sS}}}{\rho_{\mathrm{sS}}}
$$

\section{The global picture: deformation, mass transfer and diffusion}

The change of mass of each species is due a priori to both transfer, i.e a physico-chemical reaction, and diffusion, namely 


$$
\frac{\delta m_{k K}}{\delta t}=\frac{\delta}{\delta t} m_{k K}^{\text {reactive }}+\frac{\delta}{\delta t} m_{k K}^{\text {diffusive }}
$$

In practice, the changes in the species of the solid phase are purely reactive, namely by transfer, through the membrane, of water and salt between the solid and fluid phases. On the other hand, the species of the fluid phase $K=$ W may also undergo mass changes by exchanges (diffusion) with the outside, i.e.,

$$
\frac{\delta}{\delta t} m_{k K}^{\text {diffusive }}=-\operatorname{div} \mathbf{M}_{k K}
$$

where $\mathbf{M}_{k K}$ is the mass flux of the species $k$ of phase $K$ through the solid skeleton, namely,

$$
\mathbf{M}_{k K}=\rho^{k K}\left(\mathbf{v}_{k K}-\mathbf{v}_{\mathrm{S}}\right),
$$

which clearly indicates that the diffusive flux is non-zero only for species of the fluid phase. Also $\delta m_{k K}^{\text {reactive }} / \delta t$, abbreviated to $\hat{\rho}^{k K}$ in the sequel, is the rate of transfer of mass density towards the species $k$ of phase $K$, see Appendix A, Eq. (A.1). Since each of these transfers concerns a single species as indicated by Fig. 2, then

$$
\hat{\rho}^{\mathrm{WS}}+\hat{\rho}^{\mathrm{wW}}=0, \quad \hat{\rho}^{\mathrm{SS}}+\hat{\rho}^{\mathrm{sW}}=0 .
$$

In absence of thermal effects, starting from the statements of balance of mass for each species, and of momentum and energy for the phases, e.g. Eringen and Ingram (1965), the Clausius-Duhem inequality for a mixture as a whole can be cast in the following form,

$$
\delta D=-\delta \Psi+\boldsymbol{\sigma}: \delta \boldsymbol{\epsilon}+\sum_{k, K} \mu_{k K}\left(\delta m_{k K}-\hat{\rho}^{k K} \delta t\right)-\sum_{k, K} \nabla \mu_{k K} \cdot \mathbf{M}_{k K} \delta t \geqslant 0 .
$$

The Clausius-Duhem inequality can be viewed as containing the sum of three contributions, $\delta D=\delta D_{1}+$ $\delta D_{2}+\delta D_{3}$, associated with mechanical work, mass transfer and diffusion. These contributions will be required to be positive individually, that is

$$
\begin{aligned}
& \delta D_{1}=-\delta \Psi+\boldsymbol{\sigma}: \delta \boldsymbol{\epsilon}+\sum_{k, K} \mu_{k K} \delta m_{k K} \geqslant 0, \\
& \delta D_{2} / \delta t=-\left(\mu_{\mathrm{wS}}-\mu_{\mathrm{wW}}\right) \hat{\rho}^{\mathrm{wS}}-\left(\mu_{\mathrm{sS}}-\mu_{\mathrm{sW}}\right) \hat{\rho}^{\mathrm{sS}} \geqslant 0, \\
& \delta D_{3} / \delta t=-\nabla \mu_{\mathrm{wW}} \cdot \mathbf{M}_{\mathrm{wW}}-\nabla \mu_{\mathrm{sW}} \cdot \mathbf{M}_{\mathrm{sW}} \geqslant 0 .
\end{aligned}
$$

If body force density $\mathbf{b}_{k \mathrm{~W}}$ and acceleration $\mathrm{d}^{k \mathrm{~W}} \mathbf{v}_{k \mathrm{~W}} / \mathrm{d} t$ are accounted for, $\nabla \mu_{k \mathrm{~W}}$ should be substituted by $\nabla \mu_{k \mathrm{~W}}-\mathbf{b}_{k \mathrm{~W}}+\mathrm{d}^{k \mathrm{~W}} \mathbf{v}_{k \mathrm{~W}} / \mathrm{d} t$ in $\delta D_{3} / \delta t$. The chemo-hyperelastic behaviour, Section 4 , will be constructed in order the first term $\delta D_{1}$ to exactly vanish. Satisfaction of the second and third inequalities leads to a generalized transfer law and a generalized diffusion law respectively, detailed in Gajo and Loret (2001). Transfer equations introduce memory effects, akin to transfer of fluid between the types of cavities in double porosity theories. They might also be viewed as a vehicle to introduce viscous effects in these otherwise rate-independent elasto-plastic constitutive equations. Generalized diffusion equations imply that matter transfers/diffuses from a phase of larger chemical potential to a phase of lower chemical potential. On one hand, this implication appears just as a generalization of Darcy's seepage law. On the other hand, a chemically induced flow might turn out to be a counterflow to usual Darcy's flow when the chemical potentials and pressures have their relative orders of magnitude reversed. Identification of diffusion coefficients in terms of measurable quantities is described in the above reference where finite element simulations of laboratory experiments are presented. 


\section{Elastic constitutive equations}

The absorption/desorption of water (and salt) into/from the solid phase introduces a chemo-mechanical coupling. On the other hand, the presence of salt in the water phase does not affect directly the mechanical behaviour of the porous medium, it just flows through. Its amount is governed by an equation of mass conservation and a flow law. Therefore, to develop the chemo-mechanical constitutive equations, we will treat the fluid phase as a whole and, temporarily, ignore its chemical composition. Therefore constitutive equations are developed for the following variables: two thermodynamic dual variables characterizing the mechanical state of each phase, namely $(\boldsymbol{\sigma}, \boldsymbol{\epsilon})$ for the solid phase and $\left(p_{\mathrm{W}}, v_{\mathrm{W}}\right)$ for the fluid phase, as many thermodynamic dual variables of chemical potential-mass content as there are species that cross the membrane, namely $\left(\mu_{k \mathrm{~S}}, m_{k \mathrm{~S}}\right), k \in \mathrm{S}^{\leftrightarrow}$. Incompressibility of the species will reduce the number of constitutive equations by one.

In view of extension to the elastic-plastic behaviour, generalized strains will henceforth be denoted by a superscript "el". Instances are strains, volume and mass contents, number of moles. Later, these entities will be decomposed into an elastic, or reversible, part and a plastic, or irreversible, part. Given a reference state, and a process which is reversible from that reference state to the current state, the elastic part of each of above entities is by convention equal to the total entity.

\subsection{Dependent and independent variables}

When the behaviour is elastic, the energy of porous medium per unit initial volume $\mathscr{W}^{\mathrm{el}}=\Psi^{\mathrm{el}}-\Psi_{\mathrm{W}}^{\mathrm{el}}$ can be viewed as the elastic energy of the porous medium for which the chemical effects in the fluid phase are disregarded. It depends on the restricted set of independent variables ${ }^{2}\left\{\epsilon^{\mathrm{el}}, v_{\mathrm{W}}^{\mathrm{el}}, m_{\mathrm{wS}}^{\mathrm{el}}, m_{\mathrm{sS}}^{\mathrm{el}}\right\}$. Indeed, with the work definition (2.5) and the Gibbs-Duhem relation (2.22), we have the following incremental decomposition of $\Psi^{\mathrm{el}}$ into $\mathscr{W}^{\mathrm{el}}$ and $\Psi_{\mathrm{W}}^{\mathrm{el}}$ :

$$
\begin{aligned}
& \delta \Psi^{\mathrm{el}}=\boldsymbol{\sigma}: \delta \boldsymbol{\epsilon}^{\mathrm{el}}+\sum_{k \in \mathrm{S}^{\hookleftarrow}} \mu_{k \mathrm{~S}} \delta m_{k \mathrm{~S}}^{\mathrm{el}}+\sum_{k \in \mathrm{W}} \mu_{k \mathrm{~W}} \delta m_{k \mathrm{~W}}, \\
& \delta \mathscr{W}^{\mathrm{el}}=\boldsymbol{\sigma}: \delta \epsilon^{\mathrm{el}}+p_{\mathrm{W}} \delta v_{\mathrm{W}}^{\mathrm{el}}+\sum_{k \in \mathrm{S}^{\hookleftarrow}} \mu_{k \mathrm{~S}} \delta m_{k \mathrm{~S}}^{\mathrm{el}} \\
& \delta \Psi_{\mathrm{W}}^{\mathrm{el}}=-p_{\mathrm{W}} \delta v_{\mathrm{W}}^{\mathrm{el}}+\sum_{k \in \mathrm{W}} \mu_{k \mathrm{~W}} \delta m_{k \mathrm{~W}} .
\end{aligned}
$$

Thus constitutive equations for the dependent variables $\left\{\boldsymbol{\sigma}, p_{\mathrm{W}}, \mu_{\mathrm{wS}}, \mu_{\mathrm{s} S}\right\}$ in terms of the independent variables $\left\{\epsilon^{\mathrm{el}}, v_{\mathrm{W}}^{\mathrm{el}}, m_{\mathrm{wS}}^{\mathrm{el}}, m_{\mathrm{sS}}^{\mathrm{el}}\right\}$ are obtained in the format:

$$
\boldsymbol{\sigma}=\frac{\partial \mathscr{W}^{\mathrm{el}}}{\partial \boldsymbol{\epsilon}^{\mathrm{el}}}, \quad p_{\mathrm{W}}=\frac{\partial \mathscr{W}^{\mathrm{el}}}{\partial v_{\mathrm{W}}^{\mathrm{el}}}, \quad \mu_{k \mathrm{~S}}=\frac{\partial \mathscr{W}^{\mathrm{el}}}{\partial m_{k \mathrm{~S}}^{\mathrm{el}}}, \quad k=\mathrm{w}, \mathrm{s} .
$$

Alternative choices in the sets of independent and dependent variables can be postulated by partial or total Legendre transforms of $\mathscr{W}^{\mathrm{el}}$.

\subsection{Incompressible species}

In the sequel, we will restrict the formulation by assuming that all species are incompressible. We shall adopt $\left\{\epsilon^{\mathrm{el}}, m_{\mathrm{wS}}^{\mathrm{el}}, m_{\mathrm{sS}}^{\mathrm{el}}\right\}$ as the set of independent variables and then the increment of elastic fluid volume

\footnotetext{
${ }^{2}$ Notice that only the arguments that vary during loadings are listed; for example, since the mass-content in clay is constant inside the solid phase, its influence is not shown explicitly in the list of arguments although it is tacitly assumed.
} 
content is given by (2.27) written in terms of elastic quantities since we require the incompressibility condition to hold in both the elastic and elastic-plastic regimes.

Hence, $\delta \mathscr{W}^{\mathrm{el}}$, Eq. (4.1), becomes

$$
\delta \mathscr{W}^{\mathrm{el}}=\overline{\boldsymbol{\sigma}}: \delta \boldsymbol{\epsilon}^{\mathrm{el}}+\bar{\mu}_{\mathrm{wS}} \delta m_{\mathrm{wS}}^{\mathrm{el}}+\bar{\mu}_{\mathrm{sS}} \delta m_{\mathrm{sS}}^{\mathrm{el}}
$$

in terms of Terzaghi's effective stress $\overline{\boldsymbol{\sigma}}$ and of the effective chemical potentials $\bar{\mu}_{k K}$,

$$
\overline{\boldsymbol{\sigma}}=\boldsymbol{\sigma}+p_{\mathrm{W}} \mathbf{I}, \quad \bar{\mu}_{k K}=\mu_{k K}-\frac{p_{\mathrm{W}}}{\rho_{k K}} .
$$

If the mass transfer does not alter the density, Eq. (2.8), equilibrium of the chemical potentials, i.e. $\mu_{k \mathrm{~S}}=\mu_{k \mathrm{~W}}$, is equivalent to equilibrium of the effective chemical potentials, i.e. $\bar{\mu}_{k \mathrm{~S}}=\bar{\mu}_{k \mathrm{~W}}$. Of course, chemical equilibrium applies only for species that can cross the membrane.

The constitutive relations take now the form,

$$
\overline{\boldsymbol{\sigma}}=\frac{\partial \mathscr{W}^{\mathrm{el}}}{\partial \boldsymbol{\epsilon}^{\mathrm{el}}}, \quad \bar{\mu}_{k \mathrm{~S}}=\frac{\partial \mathscr{W}^{\mathrm{el}}}{\partial m_{k \mathrm{~S}}^{\mathrm{el}}}, \quad k=\mathrm{w}, \mathrm{s} .
$$

Notice that, when there is only a single species present per phase as in the usual poro-elasticity, that is, $\delta m_{\mathrm{wS}}^{\mathrm{el}}=\delta m_{\mathrm{sS}}^{\mathrm{el}}=0$, the classical relations are retrieved. Indeed, the incremental energy reduces to $\boldsymbol{\sigma}: \delta \epsilon^{\mathrm{el}}+$ $p_{\mathrm{W}} \delta v_{\mathrm{W}}^{\mathrm{el}}$ in the compressible case. In the incompressible case, the incremental energy is equal to $\overline{\boldsymbol{\sigma}}: \delta \boldsymbol{\epsilon}^{\mathrm{el}}$ since then the incompressibility condition is $\delta v_{\mathrm{W}}^{\mathrm{el}}=\delta \operatorname{tr} \epsilon^{\mathrm{el}}$ and the remaining constitutive equation (4.5) 1 specifies the effective stress only, that is the pore pressure $p_{\mathrm{W}}$ does not enter explicitly the constitutive equations: in a boundary value problem, it is defined by boundary conditions.

\subsection{Logarithmic isotropic hyperelasticity}

Experimental data over a large range of stress show that the elastic bulk moduli of soils depend on the stress state. A usual approximation linked to the Cam-Clay models consists in assuming the volumetric elastic strain to be proportional to the logarithm of the mean-effective stress. On the other hand, Bolt (1956) has shown the influence of the chemical composition of pore water on the isotropic rebound (unloading) curves from high stresses: in the plane $(\ln \bar{p}$-void ratio $e)$, they are almost linear trajectories emanating from a restricted zone with a very narrow range of void ratios. In our formulation, a constant chemical composition of the pore water is equivalent, if the pore pressure is constant, to a constant chemical potential of any species in fluid phase, and to a constant chemical potential of any species that can cross the membrane. Thus, one would be lead a priori to use the effective stress and chemical potentials as primary variables. However, the extension to electrolyte solutions is facilitated if the effective stress and the mass of species in the solid phase $m_{k \mathrm{~S}}^{\mathrm{el}}$ (or the molar fraction $x_{k \mathrm{~S}}^{\mathrm{el}}$ derived consistently from the latter according to Eq. (2.16)) are considered as primary variables instead. From a practical point of view, the above-mentioned unloading curves can be associated with an almost constant $x_{k \mathrm{~S}}^{\mathrm{el}}$ as mechanical loading, at constant chemical potential $\mu_{k \mathrm{~S}}$, will be shown to have a small influence on the chemical composition of the solid phase.

Before introducing logarithmic hyperelasticity in this chemo-mechanical context, let us first consider the purely mechanical and purely chemical contributions to the chemo-elastic potential independently. For this purpose, let us introduce the decomposition of the strain and total and effective stress tensors into their spherical and deviatoric parts, namely

$$
\boldsymbol{\epsilon}^{\mathrm{el}}=\frac{\operatorname{tr} \epsilon^{\mathrm{el}}}{3} \mathbf{I}+\operatorname{dev} \boldsymbol{\epsilon}^{\mathrm{el}}, \quad \boldsymbol{\sigma}=-p \mathbf{I}+\mathbf{s}, \quad \overline{\boldsymbol{\sigma}}=-\bar{p} \mathbf{I}+\mathbf{s}
$$

with the total and effective mean-stresses $p, \bar{p}$ and deviatoric stress $q$, 


$$
p=-\frac{\operatorname{tr} \boldsymbol{\sigma}}{3}, \quad \bar{p}=-\frac{\operatorname{tr} \bar{\sigma}}{3}, \quad q=\left(\frac{3}{2} \mathbf{s}: \mathbf{s}\right)^{1 / 2} .
$$

For chemically inert soils, the logarithmic stress-based formulation starts by assuming the stored energy in the following form,

$$
\kappa \bar{p}\left(\ln \left(\frac{\bar{p}}{\bar{p}_{0}}\right)-1\right)+\frac{q^{2}}{6 G},
$$

where $\kappa$ and $G$ are material constants and $\bar{p}_{0}$ a reference mean-stress. The corresponding secant bulk modulus and tangent bulk modulus,

$$
\frac{\delta \operatorname{tr} \overline{\boldsymbol{\sigma}} / 3}{\delta \operatorname{tr} \boldsymbol{\epsilon}^{\mathrm{el}}}=\frac{\bar{p}-\bar{p}_{0}}{\kappa \ln \left(\bar{p} / \bar{p}_{0}\right)}>0, \quad \frac{\delta \operatorname{tr} \overline{\boldsymbol{\sigma}} / 3}{\delta \operatorname{tr} \epsilon^{\mathrm{el}}}=\frac{\bar{p}}{\kappa}>0,
$$

are thus increasing with $\bar{p}$.

Let us consider now the purely chemical contribution to the chemo-elastic potential, namely $R T /$ $V_{0} \varphi\left(x_{\mathrm{wS}}^{\mathrm{el}}, x_{\mathrm{sS}}^{\mathrm{el}}\right)$. We require the chemical part of the chemical potentials $\bar{\mu}_{k \mathrm{~S}}$ to have the classic form (2.7), that is since $N_{k K}^{\mathrm{el}}=m_{k K} V_{0} / m_{k}^{(\mathrm{M})}$, we require $\partial \varphi / \partial N_{k \mathrm{~S}}^{\mathrm{el}}=\ln x_{k \mathrm{~S}}^{\mathrm{el}}, k=\mathrm{w}, \mathrm{s}$. Then up to a constant depending on the number of moles of solid particles $N_{\mathrm{cS}}$,

$$
\varphi\left(x_{\mathrm{wS}}^{\mathrm{el}}, x_{\mathrm{SS}}^{\mathrm{el}}\right)=\sum_{k \in \mathrm{S}^{\hookrightarrow}} N_{k \mathrm{~S}}^{\mathrm{el}} \ln N_{k \mathrm{~S}}^{\mathrm{el}}-\left(\sum_{k \in \mathrm{S}} N_{k \mathrm{~S}}^{\mathrm{el}}\right) \ln \left(\sum_{l \in \mathrm{S}} N_{l \mathrm{~S}}^{\mathrm{el}}\right) .
$$

We are now in the position to obtain the chemo-elastic potential in the above motivated mixed form. For that purpose, let us first define a partial Legendre transform $\mathscr{W}_{M}^{\mathrm{el}}\left(\overline{\boldsymbol{\sigma}}, m_{\mathrm{wS}}^{\mathrm{el}}, m_{\mathrm{sS}}^{\mathrm{el}}\right)$ of the energy $\mathscr{W}^{\mathrm{el}}$, namely

$$
\delta \mathscr{W}_{M}^{\mathrm{el}}=\delta\left(\overline{\boldsymbol{\sigma}}: \epsilon^{\mathrm{el}}-\mathscr{W}^{\mathrm{el}}\right)=\epsilon^{\mathrm{el}}: \delta \overline{\boldsymbol{\sigma}}-\sum_{k=\mathrm{w}, \mathrm{s}} \bar{\mu}_{k \mathrm{~S}} \delta m_{k \mathrm{~S}}^{\mathrm{el}}
$$

from which follow the constitutive relations,

$$
\boldsymbol{\epsilon}^{\mathrm{el}}=\frac{\partial \mathscr{W}_{M}^{\mathrm{el}}}{\partial \overline{\boldsymbol{\sigma}}}, \quad \bar{\mu}_{k \mathrm{~S}}=-\frac{\partial \mathscr{W}_{M}^{\mathrm{el}}}{\partial m_{k \mathrm{~S}}^{\mathrm{el}}}, \quad k=\mathrm{w}, \mathrm{s} .
$$

Let us consider now the case of a membrane permeable to water only. The remaining conjugate chemical variables are $m_{\mathrm{wS}}^{\mathrm{el}}$ and $\bar{\mu}_{\mathrm{wS}}$. In order to reproduce the expected elastic behaviour, Fig. 3, we set

$$
\mathscr{W}_{M}^{\mathrm{el}}\left(\overline{\boldsymbol{\sigma}}, m_{\mathrm{wS}}^{\mathrm{el}}\right)=-\bar{p} \operatorname{tr} \epsilon_{\kappa}^{\mathrm{el}}+\kappa\left(x_{\mathrm{wS}}^{\mathrm{el}}\right) F\left(\bar{p}, \bar{p}_{\kappa}\right)+\frac{q^{2}}{6 G}-\frac{R T}{V_{0}} \varphi\left(x_{\mathrm{wS}}^{\mathrm{el}}\right) .
$$

Here $F\left(\bar{p}, \bar{p}_{\kappa}\right)$ is the function that introduces a logarithmic dependance in mean-stress,

$$
F\left(\bar{p}, \bar{p}_{\kappa}\right)=\bar{p} \ln \frac{\bar{p}}{\bar{p}_{\kappa}}-\bar{p}, \quad \frac{\mathrm{d} F}{\mathrm{~d} \bar{p}}=\ln \frac{\bar{p}}{\bar{p}_{\kappa}}
$$

and $\operatorname{tr} \epsilon_{\kappa}^{\mathrm{el}}$ is the value of the volume change when the effective mean-stress varies from the convergence stress $\bar{p}_{\kappa}$ to a small reference value $\bar{p}_{0}$ while the pore fluid is distilled water, namely Fig. 3,

$$
\operatorname{tr} \epsilon_{\kappa}^{\mathrm{el}}=-\kappa^{\mathrm{dw}} \ln \frac{\bar{p}_{\kappa}}{\bar{p}_{0}} \quad \text { with } \kappa^{\mathrm{dw}}=\kappa\left(x_{\mathrm{wS}}^{\mathrm{el}, \mathrm{dw}}\right),
$$

where $x_{\mathrm{wS}}^{\mathrm{el}, \mathrm{dw}}$ is the elastic molar fraction of absorbed water when pore water is distilled. In absence of precise experimental influence of the chemical composition of pore water on the shear modulus $G$, the latter will be 

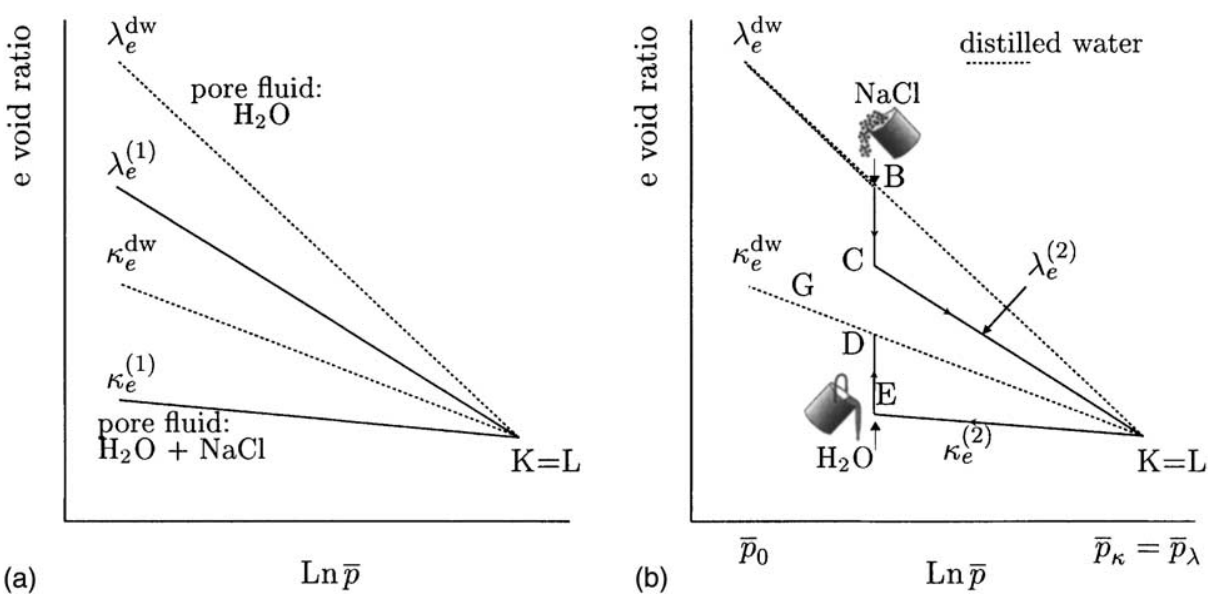

Fig. 3. In the simplified model, a sample keeps approximatively its chemical composition during a purely mechanical loading cycle and behaves like a Cam-Clay material. Chemical loading and unloading occurs in an elastic regime: after a chemical cycle, the final point $\mathrm{G}$ is back on the initial curve, corresponding here to a sample in contact with distilled water. Increase of salt concentration up to solution saturation BC leads to chemical consolidation while later re-exposure to distilled water ED displays swelling. (a) A mechanical loading cycle on a sample in contact with distilled water and on another sample in contact with a saline solution. (b) Idealized mixed mechanical and chemical cycle. The purely mechanical experiment with distilled water as a pore fluid is shown for comparison.

simply assumed to be constant. Then, in view of the elastic potential (4.13), the elastic constitutive Eq. (4.12) reduce to:

$$
\begin{aligned}
& \boldsymbol{\epsilon}^{\mathrm{el}}=\left(\operatorname{tr} \boldsymbol{\epsilon}_{\kappa}^{\mathrm{el}}-\kappa\left(x_{\mathrm{wS}}^{\mathrm{el}}\right) \ln \frac{\bar{p}}{\bar{p}_{\kappa}}\right) \frac{\mathbf{I}}{3}+\frac{\mathbf{s}}{2 G}, \\
& \bar{\mu}_{\mathrm{wS}}=-F\left(\bar{p}, \bar{p}_{\kappa}\right) \frac{\mathrm{d} \kappa}{\mathrm{d} m_{\mathrm{wS}}^{\mathrm{el}}}+\frac{R T}{m_{\mathrm{w}}^{(\mathrm{M})}} \ln x_{\mathrm{wS}}^{\mathrm{el}} .
\end{aligned}
$$

Although the formulation is by no means restricted to such states, the manipulations will become more familiar to Cam-Clay users if the deviatoric stress and strain are assumed to maintain fixed directions during loading, e.g. as for triaxial compression or extension paths, or more generally paths with constant Lode angle. Then the stress and strain states can be fully characterized by only two work-conjugate invariants, namely $(\bar{p}, q)$ and $\left(\operatorname{tr} \epsilon^{\mathrm{el}}, \epsilon_{q}^{\mathrm{el}}\right)$, since then the incremental work $\overline{\boldsymbol{\sigma}}: \delta \epsilon^{\mathrm{el}}$ reduces to $\bar{p} \delta \operatorname{tr} \epsilon^{\mathrm{el}}+q \delta \epsilon_{q}^{\mathrm{el}}$. An incremental form of the elastic constitutive equations giving $\left(-\delta \bar{p}, \delta q, \delta \bar{\mu}_{\mathrm{wS}}\right)$ in terms of $\left(\delta \operatorname{tr} \epsilon, \delta \epsilon_{q}^{\mathrm{el}}, \delta m_{\mathrm{wS}}^{\mathrm{el}}\right)$ can be expressed via the symmetric matrix,

$$
\left[\begin{array}{c}
-\delta \bar{p} \\
\delta q \\
\delta \bar{\mu}_{\mathrm{wS}}
\end{array}\right]=\left[\begin{array}{ccc}
B & 0 & B_{\mathrm{w}} \\
0 & 3 G & 0 \\
B_{\mathrm{w}} & 0 & \beta
\end{array}\right]\left[\begin{array}{c}
\delta \operatorname{tr} \epsilon^{\mathrm{el}} \\
\delta \epsilon_{q}^{\mathrm{el}} \\
\delta m_{\mathrm{wS}}^{\mathrm{el}}
\end{array}\right],
$$

where the tangent bulk modulus $B$ and the coefficients $B_{\mathrm{w}}$ and $\beta$ are defined as follows:

$$
B=\frac{\bar{p}}{\kappa\left(x_{\mathrm{wS}}^{\mathrm{el}}\right)}, \quad B_{\mathrm{w}}=-\frac{\partial \bar{p}}{\partial m_{\mathrm{wS}}^{\mathrm{el}}}=\frac{\partial \bar{\mu}_{\mathrm{wS}}}{\partial \operatorname{tr} \epsilon^{\mathrm{el}}}=B \ln \frac{\bar{p}}{\bar{p}_{\kappa}} \frac{\mathrm{d} \kappa}{\mathrm{d} m_{\mathrm{wS}}^{\mathrm{el}}}
$$

and, using relation (2.17) for $k=l=\mathrm{w}$ applied to elastic entities,

$$
\beta=\frac{\partial \bar{\mu}_{\mathrm{wS}}}{\partial m_{\mathrm{wS}}^{\mathrm{el}}}=\frac{B_{\mathrm{w}}^{2}}{B}-F\left(\bar{p}, \bar{p}_{\kappa}\right) \frac{\mathrm{d}^{2} \kappa}{\mathrm{d}^{2} m_{\mathrm{wS}}^{\mathrm{el}}}+\frac{R T}{m_{\mathrm{w}}^{(\mathrm{M})}} \frac{1-x_{\mathrm{wS}}^{\mathrm{el}}}{m_{\mathrm{wS}}^{\mathrm{el}}} .
$$


Notice that the symmetry of the constitutive matrix in (4.17) is due to the existence of the chemo-elastic potential. Moreover, since $G$ is independent of the chemical composition of the solution, there is no coupling between shear components and the chemical variable.

The calibration of a typical interpolation function for $\kappa\left(x_{\mathrm{wS}}^{\mathrm{el}}\right)$ is provided in Appendix $\mathrm{B}$, with data corresponding to distilled and salt-saturated pore water.

\section{Elastic-plastic constitutive equations}

Exchange of matter between the clay cluster (solid phase) and pore water (fluid phase) implies changes in the thermomechanical state of the porous medium. Both elastic and elastic-plastic properties are affected. Chemical effects act reversibly on the plastic properties by increasing or decreasing the preconsolidation stress and also by changing the shear-strength. But by the same token, they also implicitly trigger or prevent plastic strains. Therefore chemical effects are, even qualitatively, different from thermal effects which essentially decrease the preconsolidation stress or from desaturation effects which increase this preconsolidation stress.

The terms elastic and plastic mass change are to be understood as reversible and irrreversible reactions, that is which can proceed in both or only one direction of transfer, in agreement with the respective meanings in mechanics of solids.

We begin by providing a simplified description of experimental data, delineating the key features of the model that will be given an analytical formulation in a second step.

\subsection{Experimental observations}

As already stated, the present analysis is devoted to clays which contain essentially one type of cation, like the Ponza bentonite studied by Di Maio (1996) which is a predominantly Na-Montmorillonite. The presence of several cations requires to account explicitly for electroneutrality and will be the subject of a companion paper, Gajo et al. (2002). The main features of the behaviour of Na-Montmorillonite that emanate from the oedometric tests of Di Maio (1996) and that we intend to model are listed now.

\subsubsection{Mechanical loading}

The specimen is in contact with a large reservoir of constant chemical composition and at atmospheric pressure, so that $p_{\mathrm{W}} \sim 0$. The load is continuously varied, sufficiently slow however that chemical equilibrium can be established at the end of each load increment. Therefore, one may assume the chemical potentials of all species that can transfer to be equal, at the end of each load increment, to the known chemical potentials of their counterparts in pore water.

Experiments show that the curves $(\ln \bar{p}$, void ratio $e)$ are nearly straight and converging to a narrow interval of void ratio, Fig. 3 (the coefficients $\kappa_{e}$ and $\lambda_{e}$ refer to the void ratio while the coefficients $\kappa$ and $\lambda$ refer to the volumetric strains). However, the slopes of the loading and unloading curves decrease as the $\mathrm{Na}$-content of the pore water increases. This trend holds whatever this content, that is from zero Nacontent (distilled water) to saturated solutions (that is at $20 \mathrm{~K}$ and under atmospheric pressure, 6.15 moles of $\mathrm{Na}$ per liter of water).

\subsubsection{Chemical loading: chemical consolidation and swelling}

Under constant mechanical conditions, a chemical loading consists in varying the Na-content of the pore water. When the latter increases, the void ratio decreases, and the decrease rate is especially large at small salt content: in fact most of the volume change occurs for Na-concentrations between 0 and $1 \mathrm{~mol} / \mathrm{l}$. This is true for both chemical consolidation and swelling according to unpublished results by Di Maio, Fig. 8(b). If 
the Na-content of pore water is decreased, either immediately at constant stress, or after a mechanical loading path, the volume of the sample increases. Notice that these volume changes are in qualitative agreement with the osmotic effect: flow occurs towards the region of higher salinity. Alternatively, one may say that water desorption/absorption to clay surfaces occurs to equilibrate the salt contents in pore water and clay pockets.

\subsubsection{Chemical softening and preconsolidation: a paradox}

Mechanical loading at constant chemical composition corresponds clearly to an elastic-plastic behaviour while mechanical unloading can be conjectured to be purely elastic, see Fig. 8 of Di Maio (1996). The situation is more complex for chemical loadings. At relatively low stresses, a chemical loading cycle seems to be practically reversible while the amount of plastic contractancy increases with the applied stress, Fig. 7.

Also, when chemical consolidation at a constant low stress is followed by mechanical loading, the slope of the stress-strain curve is the elastic-plastic slope right from the beginning of the stress increase, so that there seems to be almost no chemical preconsolidation, Fig. 6(e) and (f). The data available do not allow to reveal if this description is still correct or not at larger stresses.

The above interpretation may have to account for the large void ratio of Ponza bentonite. Other tests on Bisaccia clay ${ }^{3}$ at smaller void ratios, Fig. 9(b), show in fact some chemical preconsolidation. This state of affairs, chemical softening leading to plasticity and chemical preconsolidation, seems somehow paradoxical. The model developed here is able to provide an explanation of these phenomena. In fact, we have

(i) a first conceptual model for which chemical loading and unloading are purely elastic, Fig. 3. In this model, loading curves have slopes $\lambda$ that depend on the chemical composition of pore water, that is equivalently, as explained above, $\lambda=\lambda\left(\bar{\mu}_{\mathrm{wS}}\right) .{ }^{4}$ They converge at a point $P_{\lambda}$, from which unloading curves emanate as well, i.e. $P_{\kappa}=P_{\lambda}$, with slopes $\kappa$ depending also of the chemical composition of pore water. This dependance has been introduced in the previous section through the elastic mass content of absorbed water $m_{\mathrm{wS}}^{\mathrm{el}}$, Eq. (4.16); alternatively, it could be introduced through its chemical potential $\bar{\mu}_{\mathrm{wS}}$. The two representations are different only in form. Quantitatively they are almost equivalent because the chemical term dominates the mechanical term in the chemical potential.

In this simplified model, the difference $\lambda-\kappa$ is constant at varying chemical pore composition. To explore the consequences of this assumption, let us consider a mixed mechanical and chemical loading and unloading path, Fig. 3(b). The chemical swelling ED is assumed elastic and a constant $\lambda-\kappa$ implies CE to be equal to $\mathrm{BD}$, and so the chemical consolidation $\mathrm{BC}$ is equal to the chemical swelling $\mathrm{ED}$, and it is therefore elastic.

(ii) a more complete model that accounts for chemical softening and preconsolidation. In this model, chemical consolidation has first an elastic-plastic stage, possibly followed by a purely elastic stage, the relative size of the former increasing with the stress level, Fig. 4(a). Moreover, the limit points $P_{\kappa}$ and $P_{\lambda}$ are a priori distinct. There are two competing effects during chemical consolidation at constant stress. Plasticity leads to plastic contractancy, that is to hardening which increases the preconsolidation stress $p_{\mathrm{c}}$. Chemical effects tend to decrease $p_{\mathrm{c}}$ up to some value of the chemical load, say in terms of the chemical potential $\bar{\mu}_{\mathrm{wS}}^{\mathrm{cr}}$, i.e. stage BE of Fig. 4(a); for a larger chemical load the behaviour is elastic since the chemical effect tends to increase $p_{\mathrm{c}}$, so that there is chemical preconsolidation, i.e. stage EC of Fig. 4(a). From the data available, namely Fig. 7 of Di Maio and Fenelli (1997), this elastic (chemical preconsolidation) stage EC seems to decrease with respect to the elastic-plastic and chemical softening stage BE at higher stresses.

\footnotetext{
${ }^{3}$ Bisaccia clay is not a Na-Montmorillonite clay. However part of its behaviour can be explained as if it were. But simulations of complex mixed mechanical and chemical loadings on that clay require to account for the presence of several cations, see Gajo et al. (2002).

${ }^{4}$ This formalism is adequate for incompressible species and a semi-permeable membrane.
} 

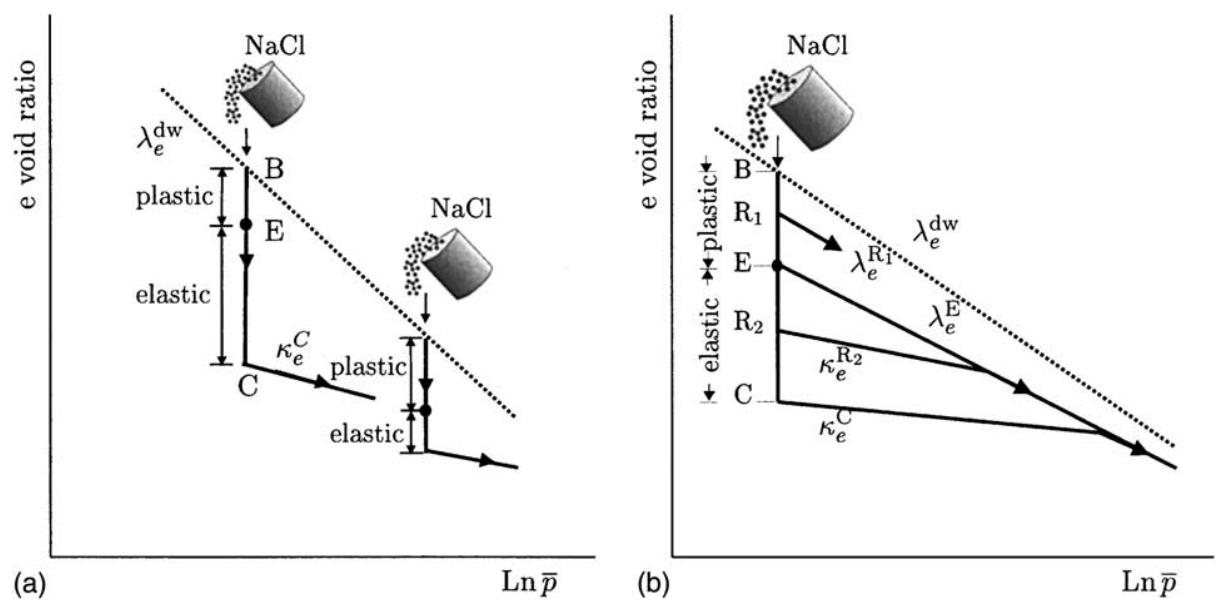

Fig. 4. Chemical loading (increase of salt concentration) occurs first plastically and later elastically. Mechanical reloading shows preconsolidation only if this second stage has been reached. (a) During chemical loading, the relative part of the plastic stage increases with the level of the effective mean-stress. The chemical loading path $\mathrm{BC}$ in this. (b) Mechanical reloading from $\mathrm{R}_{1}$ in the plastic stage displays a plastic behaviour. Reloading from $\mathrm{R}_{2}$ in the elastic stage shows an elastic behaviour and reveals chemical preconsolidation.

Let us describe now the expected behaviour upon resuming mechanical loading, in the context of this more complete model:

- if mechanical loading resumes during the elastic-plastic stage, point $\mathrm{R}_{1}$ between points $\mathrm{B}$ and $\mathrm{E}$ on Fig. 4(b), the behaviour will continue to be elastic-plastic following the current $\lambda\left(\bar{\mu}_{\mathrm{wS}}\right)$-line.

- if mechanical loading resumes during the elastic stage, point $\mathrm{R}_{2}$ below point $\mathrm{E}$ on Fig. 4(b), the behaviour will be elastic, following the current $\kappa\left(\bar{\mu}_{\mathrm{wS}}\right)$-line, up to the point where the $\lambda\left(\bar{\mu}_{\mathrm{wS}}^{\mathrm{E}}\right)$-line is reached.

Let us consider now a chemical load cycle, Fig. 5. The final void ratio, point $\mathrm{D}$, is below the initial void ratio, point $\mathrm{B}$, due to the plastic contractancy during chemical consolidation BE, Fig. 5(a). Mechanical loading from point $\mathrm{D}$ will show an elastic behaviour since the negative chemical effect is more than compensated by the positive plastic hardening which has kept the value it has acquired during loading at point E: Fig. 5(c) indicates the evolution of $p_{\mathrm{c}}$ during chemical unloading.

The qualitative behaviour described above is now given an analytic expression.

\subsection{Incremental elastic-plastic relations}

\subsubsection{The general case of compressible species and imperfect membrane}

The generalized strains are endowed with an elastic part (superscript 'el') and a plastic part (superscript 'pl'), namely

$$
\boldsymbol{\epsilon}=\boldsymbol{\epsilon}^{\mathrm{el}}+\boldsymbol{\epsilon}^{\mathrm{pl}}, \quad v_{\mathrm{W}}=v_{\mathrm{W}}^{\mathrm{el}}+v_{\mathrm{W}}^{\mathrm{pl}}, \quad m_{k \mathrm{~S}}=m_{k \mathrm{~S}}^{\mathrm{el}}+m_{k \mathrm{~S}}^{\mathrm{pl}}, \quad k=\mathrm{w}, \mathrm{s} .
$$

The elastic components in this expression are expressed through (4.12), (4.16) or (4.17). The plastic incremental flow relations are motivated by the dissipation inequality (3.6). In fact, using the hyperelastic relations and the strain decomposition (5.1), the resulting dissipation, $\delta D_{1}$, can be cast in the following format, 

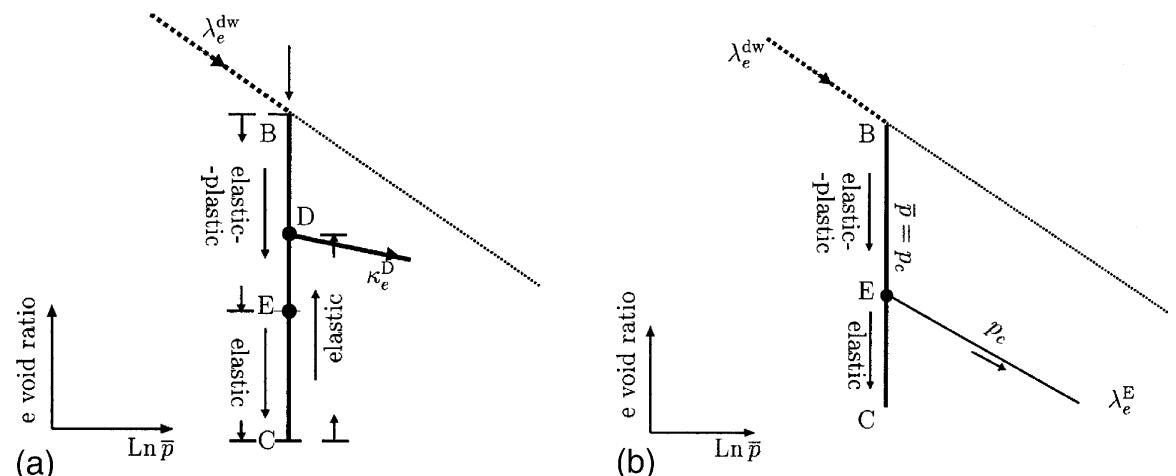

(b)

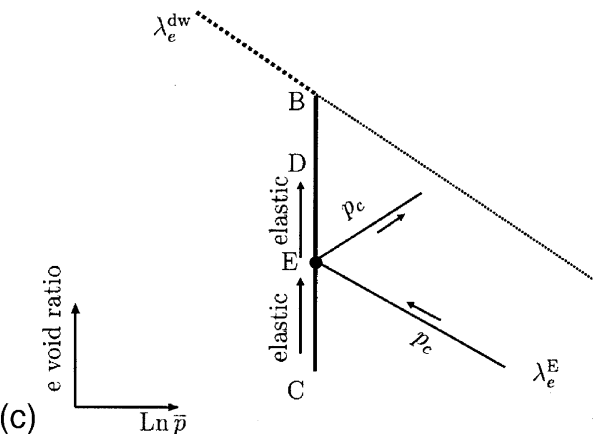

Fig. 5. Evolution of preconsolidation stress during a chemical loading cycle. Chemical loading is first elastic-plastic, next elastic while chemical unloading is elastic: so the strain-induced preconsolidation remains fixed on the paths EC and CED. The preconsolidation stress is affected both reversibly and irreversibly by chemical content. (a) Chemical loading $\mathrm{BC}$ and unloading cycle $\mathrm{CD}$, that is progressive salinization and desalinization of pore water. (b) Evolution of preconsolidation stress $p_{\mathrm{c}}$ during chemical loading $\mathrm{BC}$. During the plastic stage $\mathrm{BE}, p_{\mathrm{c}}=\bar{p}$ as there is competition between plastic hardening and chemical softening. In the elastic stage EC, chemical hardening produces preconsolidation. (c) Evolution of preconsolidation stress $p_{\mathrm{c}}$ during chemical unloading CD. During desalinization of pore water, chemical strengthening is reversibly wiped out until point $\mathrm{E}$ is reached. Upon further unloading, the previously chemical softening reverses sign and becomes chemical hardening.

$$
\delta D_{1}=\boldsymbol{\sigma}: \delta \epsilon^{\mathrm{pl}}+p_{\mathrm{W}} \delta v_{\mathrm{W}}^{\mathrm{pl}}+\sum_{k \in \mathrm{S}^{\hookleftarrow}} \mu_{k \mathrm{~S}} \delta m_{k \mathrm{~S}}^{\mathrm{pl}} \geqslant 0,
$$

which motivates the generalized normality flow rule,

$$
\delta \epsilon^{\mathrm{pl}}=\delta \Lambda \frac{\partial g}{\partial \boldsymbol{\sigma}}, \quad \delta v_{\mathrm{W}}^{\mathrm{pl}}=\delta \Lambda \frac{\partial g}{\partial p_{\mathrm{W}}}, \quad \delta m_{k \mathrm{~S}}^{\mathrm{pl}}=\delta \Lambda \frac{\partial g}{\partial \mu_{k \mathrm{~S}}}, \quad k=\mathrm{w}, \mathrm{s},
$$

where $\delta \Lambda \geqslant 0$ is the plastic multiplier and $g=g\left(\boldsymbol{\sigma}, p_{\mathrm{W}}, \mu_{\mathrm{wS}}, \mu_{\mathrm{sS}}\right)$ the plastic potential.

\subsubsection{Incompressible species and perfect semi-permeable membrane}

Henceforth, the analysis is restricted to incompressible species and to a membrane permeable to water only. The incompressibility constraint (2.27), that holds in both the elastic and elastic-plastic regimes, provides the increment of plastic volume change of the fluid phase,

$$
\delta v_{\mathrm{W}}^{\mathrm{pl}}=\delta \operatorname{tr} \epsilon^{\mathrm{pl}}-\frac{\delta m_{\mathrm{wS}}^{\mathrm{pl}}}{\rho_{\mathrm{wS}}}-\frac{\delta m_{\mathrm{sS}}^{\mathrm{pl}}}{\rho_{\mathrm{sS}}} .
$$


The dissipation inequality (5.2) becomes

$$
\delta D_{1}=\overline{\boldsymbol{\sigma}}: \delta \boldsymbol{\epsilon}^{\mathrm{pl}}+\bar{\mu}_{\mathrm{wS}} \delta m_{\mathrm{wS}}^{\mathrm{pl}}=-\bar{p} \operatorname{tr} \delta \epsilon^{\mathrm{pl}}+\mathbf{s}: \operatorname{dev} \delta \boldsymbol{\epsilon}^{\mathrm{pl}}+\bar{\mu}_{\mathrm{wS}} \delta m_{\mathrm{wS}}^{\mathrm{pl}} \geqslant 0 .
$$

If, for simplicity, one restricts the analysis to stress paths with constant Lode angles, that expression motivates the generalized normality flow rule

$$
\operatorname{tr} \delta \epsilon^{\mathrm{pl}}=-\delta \Lambda \frac{\partial g}{\partial \bar{p}}, \quad \operatorname{dev} \delta \epsilon^{\mathrm{pl}}=\delta \Lambda \frac{\partial g}{\partial q} \frac{3}{2} \frac{\mathbf{s}}{q}\left(\Rightarrow \delta \epsilon_{q}^{\mathrm{pl}}=\delta \Lambda \frac{\partial g}{\partial q}\right), \quad \delta m_{\mathrm{wS}}^{\mathrm{pl}}=\delta \Lambda \frac{\partial g}{\partial \bar{\mu}_{\mathrm{wS}}} .
$$

Notice that in general the plastic increment of volume change of the fluid phase (5.4) has no reason to vanish: in fact, it has been introduced specifically in order the incompressibility condition be satisfied. Its existence in the compressible case allows a smooth transition between compressible and incompressible materials.

The plastic potential $g$ and consequently the yield function $f$ depend on the three arguments $\bar{p}, q, \bar{\mu}_{\mathrm{wS}}$, to which we add $\operatorname{tr} \epsilon^{\mathrm{pl}}$, which will allow hardening or softening. When there is plastic loading, that is the stress point is on the yield surface and stays there, $f=0$ and $\delta f=0$, the incremental constitutive equations become,

$$
\left[\begin{array}{c}
-\delta \bar{p} \\
\delta q \\
\delta \bar{\mu}_{\mathrm{wS}}
\end{array}\right]=\left[\begin{array}{ccc}
B-\frac{1}{H} g_{p} f_{p} & -\frac{1}{H} g_{p} f_{q} & B_{w}-\frac{1}{H} g_{p} f_{\mathrm{w}} \\
-\frac{1}{H} g_{q} f_{p} & 3 G-\frac{1}{H} g_{q} f_{q} & -\frac{1}{H} g_{q} f_{\mathrm{w}} \\
B_{\mathrm{w}}-\frac{1}{H} g_{\mathrm{w}} f_{p} & -\frac{1}{H} g_{\mathrm{w}} f_{q} & \beta-\frac{1}{H} g_{\mathrm{w}} f_{\mathrm{w}}
\end{array}\right]\left[\begin{array}{c}
\delta \operatorname{tr} \boldsymbol{\epsilon} \\
\delta \epsilon_{q} \\
\delta m_{\mathrm{wS}}
\end{array}\right] .
$$

The general expressions of the coefficients entering in (5.7), namely $f_{p}, f_{q}, f_{\mathrm{w}}, g_{p}, g_{q}, g_{\mathrm{w}}$, of the plastic modulus $H>0$ and of the hardening modulus $h$ are reported in Appendix C, together with their specializations when the yield function and plastic potential are of the Modified Cam-Clay type, namely

$$
f=f\left(\bar{p}, q, \bar{\mu}_{\mathrm{wS}}, \operatorname{tr} \epsilon^{\mathrm{pl}}\right)=\frac{q^{2}}{M^{2} \bar{p}}+\bar{p}-p_{\mathrm{c}},
$$

with $M=M\left(\bar{\mu}_{\mathrm{wS}}\right)$ and $p_{\mathrm{c}}=p_{\mathrm{c}}\left(\bar{\mu}_{\mathrm{wS}}, \operatorname{tr} \epsilon^{\mathrm{pl}}\right)$. Notice that the major symmetry of the elastic-plastic incremental relations holds iff the flow rule is associative, namely $f=g$.

The calibrations of typical interpolation functions for $\lambda\left(\bar{\mu}_{\mathrm{wS}}\right)$ and $M=M\left(\bar{\mu}_{\mathrm{wS}}\right)$ are provided in Appendix $\mathrm{D}$, with data corresponding to distilled and salt-saturated pore water.

\subsubsection{Mechanical and chemical hardening/softening}

To close the model, the influence of absorbed water on the preconsolidation stress $p_{\mathrm{c}}$ is now addressed. The more complete model is described first, as the simplified model will be just a particular case.

The consolidation lines have a slope $\lambda\left(\bar{\mu}_{\mathrm{wS}}\right)$ and they converge to the effective mean-stress $\bar{p}_{\lambda}$,

$$
\operatorname{tr} \epsilon=\operatorname{tr} \epsilon_{\lambda}-\lambda\left(\bar{\mu}_{\mathrm{wS}}\right) \ln \frac{\bar{p}}{\bar{p}_{\lambda}}, \quad \operatorname{tr} \epsilon_{\lambda}=-\lambda^{\mathrm{dw}} \ln \frac{\bar{p}_{\lambda}}{\bar{p}_{0}}
$$

while the unloading curves emanate a priori from a different point $\bar{p}_{k}$, Eqs. (4.15) and (4.16),

$$
\operatorname{tr} \epsilon^{\mathrm{el}}=\operatorname{tr} \epsilon_{\kappa}^{\mathrm{el}}-\kappa\left(x_{\mathrm{wS}}^{\mathrm{el}}\right) \ln \frac{\bar{p}}{\bar{p}_{\kappa}}, \quad \operatorname{tr} \epsilon_{\kappa}^{\mathrm{el}}=-\kappa^{\mathrm{dw}} \ln \frac{\bar{p}_{\kappa}}{\bar{p}_{0}},
$$

with $\lambda^{\mathrm{dw}}=\lambda\left(\bar{\mu}_{\mathrm{wS}}^{\mathrm{dw}}\right), \kappa^{\mathrm{dw}}=\kappa\left(x_{\mathrm{wS}}^{\mathrm{eld}, \mathrm{dw}}\right)$. Combining (5.9) and (5.10) provides the preconsolidation stress $p_{\mathrm{c}}$. In order to reduce the chemical dependance of $p_{\mathrm{c}}$ to a single parameter, namely $\bar{\mu}_{\mathrm{wS}}$, we introduce a slight qualitative modification, expressing the chemical dependance of $\kappa$ in terms of $\bar{\mu}_{\mathrm{wS}}$, namely $\kappa=\tilde{\kappa}\left(\bar{\mu}_{\mathrm{wS}}\right)$. The resulting preconsolidation stress $p_{\mathrm{c}}$ given implicitly in the following format: 


$$
(\lambda-\tilde{\kappa}) \ln \frac{p_{\mathrm{c}}}{\bar{p}_{0}}=-\operatorname{tr} \epsilon^{\mathrm{pl}}+\left((\lambda-\tilde{\kappa})-\left(\lambda^{\mathrm{dw}}-\tilde{\kappa}^{\mathrm{dw}}\right)\right) \ln \frac{\bar{p}_{\lambda}}{\bar{p}_{0}}+\left(\tilde{\kappa}-\tilde{\kappa}^{\mathrm{dw}}\right) \ln \frac{\bar{p}_{\lambda}}{\bar{p}_{\kappa}}
$$

with differential

$$
(\lambda-\tilde{\kappa}) \frac{\delta p_{\mathrm{c}}}{p_{\mathrm{c}}}=-\delta \operatorname{tr} \epsilon^{\mathrm{pl}}+\ln \frac{\bar{p}_{\lambda}}{p_{\mathrm{c}}} \delta \lambda-\ln \frac{\bar{p}_{\kappa}}{p_{\mathrm{c}}} \delta \tilde{\kappa},
$$

appears as a generalization of the usual Cam-Clay expression.

Implicit in the above description is the assumption that plasticity occurs first during the chemical loading. This requires that the initial value of the chemical contribution to $p_{\mathrm{c}}$, Eq. (5.12), be negative. In the example detailed later, this condition will imply some relations between the chemical variations of $\tilde{\kappa}$ and $\lambda$, see Appendix D, namely between the initial slopes $\kappa_{3}$ and $\lambda_{3}$. If, on the other hand, one were assuming that elasticity occurs first followed by plasticity, then preconsolidation would be excluded at saturation, a situation which is not impossible a priori for some clays but that does not correspond to the experimental behaviour of Bisaccia clay as described in Section 6.

Remark 5.1. The simplified model without chemical preconsolidation.

When the difference $\lambda-\kappa$ is constant and the effective mean-stresses $\bar{p}_{\kappa}$ and $\bar{p}_{\lambda}$ are equal, the preconsolidation stress $p_{\mathrm{c}}$, Eqs. (5.11) and (5.12), does not depend on the chemical effects. Chemical loadings and unloadings are then elastic, Fig. 3. Furthermore, the elastic-plastic incremental response to isotropic paths simplifies, since then $f$ and $g$ do not depend on $\bar{\mu}_{\mathrm{wS}}: \delta q=0, \delta \epsilon_{q}=0$, and, for the associative Modified CamClay (5.8),

$$
\left[\begin{array}{c}
-\delta \bar{p} \\
\delta \bar{\mu}_{\mathrm{wS}}
\end{array}\right]=\frac{1}{\lambda}\left[\begin{array}{cc}
\bar{p} & B_{\mathrm{w}} \kappa \\
B_{\mathrm{w}} \kappa & \beta \lambda-\frac{B_{\mathrm{w}}^{2}}{B}(\lambda-\kappa)
\end{array}\right]\left[\begin{array}{c}
\delta \operatorname{tr} \epsilon \\
\delta m_{\mathrm{wS}}
\end{array}\right]
$$

\section{Simulations of mechanical, chemical and mixed loading paths}

The subsequent simulations aim at quantifying the capacity of the model to capture the main features of the chemo-mechanical coupling that have been described in the previous sections. For that purpose, it would be necessary to have available a series of experiments on homogeneous specimens. Unfortunately, it proves difficult either to prepare artificial specimens or to get in situ specimens that can be considered homogeneous, especially in view of the complex microstructural compositions of sensitive clays. Specimens from a single borehole but taken at different depths may differ considerably even if they belong to the same soil layer. Another key difficulty stems from the time duration necessary to perform chemical loading for which chemical equilibrium can be considered to hold at any time. Only steady states at which the concentrations are deemed uniform throughout the specimen are considered. An order of magnitude of the duration the physico-chemical processes need to reach equilibrium can be grasped from the chemical tests at fixed mean-stresses shown in Fig. 7(b). They refer to oedometric tests on cylindrical specimens of initial height $20 \mathrm{~mm}$. Chemical consolidation appears to reach equilibrium in few days, while swelling is a much slower process, requiring several weeks. The experimental data used here are assumed to represent effectively a succession of equilibrium states, i.e.

$$
\bar{\mu}_{k \mathrm{~S}}=\bar{\mu}_{k \mathrm{~W}} \quad \forall k \in \mathrm{S}^{\leftrightarrow} .
$$

The validity of the above assumptions of homogeneity and equilibrium is critically examined in a subsequent finite element analysis, Gajo and Loret (2001). 


\subsection{Material parameters}

The main source of data that we have used concerns clays of Southern Italy, Di Maio (1996), Di Maio and Fenelli (1997) and Di Maio and Onorati (1999). As mentioned previously, one of the clays analyzed by these authors, Ponza bentonite, is an almost pure Na-Montmorillonite which fits in the present analysis. Part of the behaviour of Bisaccia clay, a natural marine-origin clay, is also explained when it is exposed to distilled water or saline solutions of $\mathrm{NaCl}$ only. Most of the experimental data have been obtained in oedometric tests; however for the sake of simplification, they are used as isotropic tests for parameter calibration and subsequent simulations. The key material parameters of these clays are reported in Table 1 and the resulting model coefficients are provided in Table 2: they have been obtained using the calibration methods described in Appendices B and D.

The material behaviour referred to as 'Ponza bentonite simplified' has been modeled using a simplified calibration, with $\kappa_{3}$ and $\lambda_{3}$, Eqs. (B.4) and (D.3), simply set equal to 1 .

The Ponza bentonite and Bisaccia clay have been remolded using distilled water and later exposed to various saline pore solutions. The mixed Bisaccia clay has been obtained by mixing clay particles with a saline solution $(1 \mathrm{~mol} / \mathrm{l}$ of $\mathrm{NaCl})$. During mechanical loading, the sample has been assumed to remain in contact with its initial pore solution; however even if it is exposed to distilled water during the mechanical loading, the duration of the triaxial test is so small that a chemical loading which involves a diffusion process has no time to take place.

Initial conditions are obtained as described in Appendix E.

\subsection{Simulations of mechanical and chemical loadings and unloadings}

In most of the simulations, the situation where the specimen is in contact with distilled water and undergoes a purely mechanical loading has been used as a reference.

Fig. 6 shows a mixed chemo-mechanical loading and unloading cycle. The mechanical load is induced by the change of effective mean-stress while the chemical load is induced by the change of molar fraction of $\mathrm{NaCl}$ in pore water. Due to the elastic-plastic behaviour that takes place during increase of the salinity of pore water (chemical loading), the final void ratio after completion of the loading cycle does not reach exactly the distilled water reference although the gap is quite small. The consolidation curves are nearly

Table 1

Initial conditions and material parameters

\begin{tabular}{lllllllll}
\hline Clay type & $e_{0}$ & $p_{\mathrm{c} 0}(\mathrm{kPa})$ & $\kappa^{\mathrm{dw}}$ & $\kappa^{\mathrm{sat}}$ & $\lambda^{\mathrm{dw}}$ & $\lambda^{\text {sat }}$ & $M^{\mathrm{dw}}$ & - \\
\hline Ponza bentonite & 8.00 & 20 & 0.081 & 0.011 & 0.171 & 0.101 & - \\
$\begin{array}{l}\text { Ponza bentonite } \\
\text { (simplified) }\end{array}$ & 8.00 & 20 & 0.081 & 0.011 & 0.180 & 0.121 & - & - \\
Bisaccia clay & & & & & & & - & - \\
Mixed Bisaccia clay & 2.24 & 10 & 0.044 & 0.009 & 0.129 & 0.094 & - \\
\hline
\end{tabular}

Table 2

Model coefficients

\begin{tabular}{lllllllll}
\hline Material & $\kappa_{1}$ & $\kappa_{3}$ & $\bar{p}_{\kappa}(\mathrm{kPa})$ & $\lambda_{1}$ & $\lambda_{3}$ & $\bar{p}_{\lambda}(\mathrm{kPa})$ & $M_{1}$ & $M_{3}$ \\
\hline Ponza bentonite & -0.070 & 6 & 1400 & -0.070 & 6 & 2600 & - & - \\
Ponza bentonite(simplified) & -0.092 & 1 & 1400 & -0.080 & 1 & 2600 & - & - \\
Bisaccia clay & -0.035 & 3 & 700 & -0.035 & 6 & 700 & - & - \\
Mixed Bisaccia clay & -0.035 & 3 & 700 & -0.061 & 6 & 700 & 0.402 & 6 \\
\hline
\end{tabular}



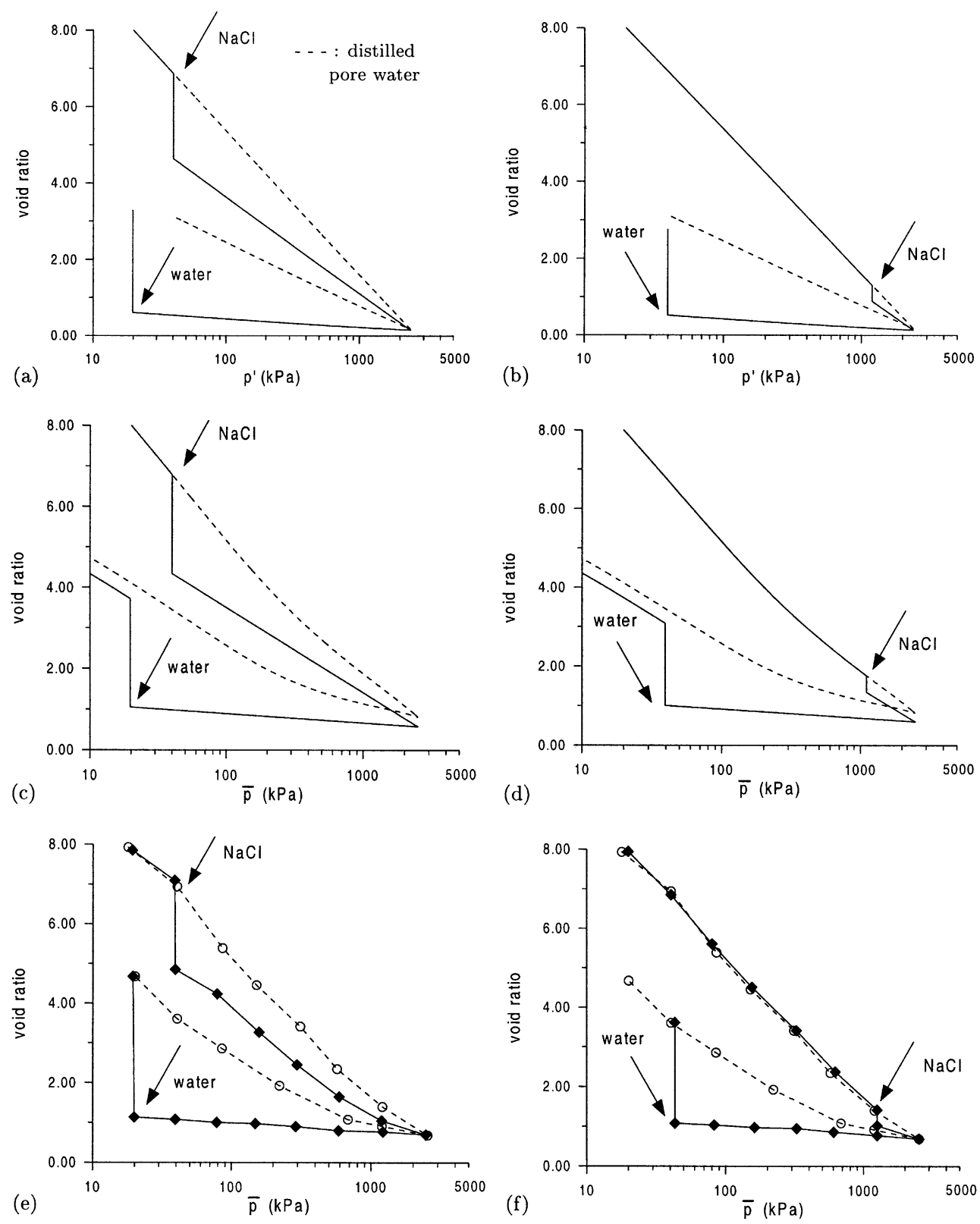

Fig. 6. Comparison of a purely mechanical loading cycle for a specimen in contact with distilled water (dashed curve) with a mixed mechanical-chemical loading cycle for a specimen initially in contact with distilled water (solid curve). The salinity of pore water (with $\mathrm{NaCl}$ ) is varied between its minimum value (distilled water) and its maximum value corresponding to saturation. Increase of salinity leads to chemical consolidation while decrease of salinity leads to swelling. Model simulations (a)-(d) and experimental data (e) and (f) on Ponza bentonite by Di Maio (1996, Fig. 8). (a) Model simulations, $\kappa_{3}=\lambda_{3}=1$; (b) model simulations, same as (a); (c) model simulations, $\kappa_{3}=\lambda_{3}=6$; (d) model simulations, same as (c); (e) experimental data; (f) experimental data.

straight in the model 'Ponza bentonite simplified', Fig. 6(a) and (b). In fact, instead of taking an average slope over the available range of mean-stresses, we have preferred to calibrate the model from the straight 
part of the mechanical compression. As a consequence, the void ratio at the end of the loading process is slightly smaller than it should. On the other hand, when the chemical dependance of $\kappa$ and $\lambda$ is more properly calibrated from the chemical consolidation test in Fig. 8, the consolidation curves tend to become less steep at large stresses, in agreement with experimental data.

\subsection{Simulations of chemical loading cycles}

Simulations of purely chemical loading cycles at given effective mean-stress are displayed in Fig. 7: plastic loading that occurs during chemical consolidation leads, at larger mean-stresses, to an increased proportion of plastic contractancy with respect to elastic swelling. This feature of the model is also displayed by experimental data. However the agreement between model simulations and experimental data seems to be
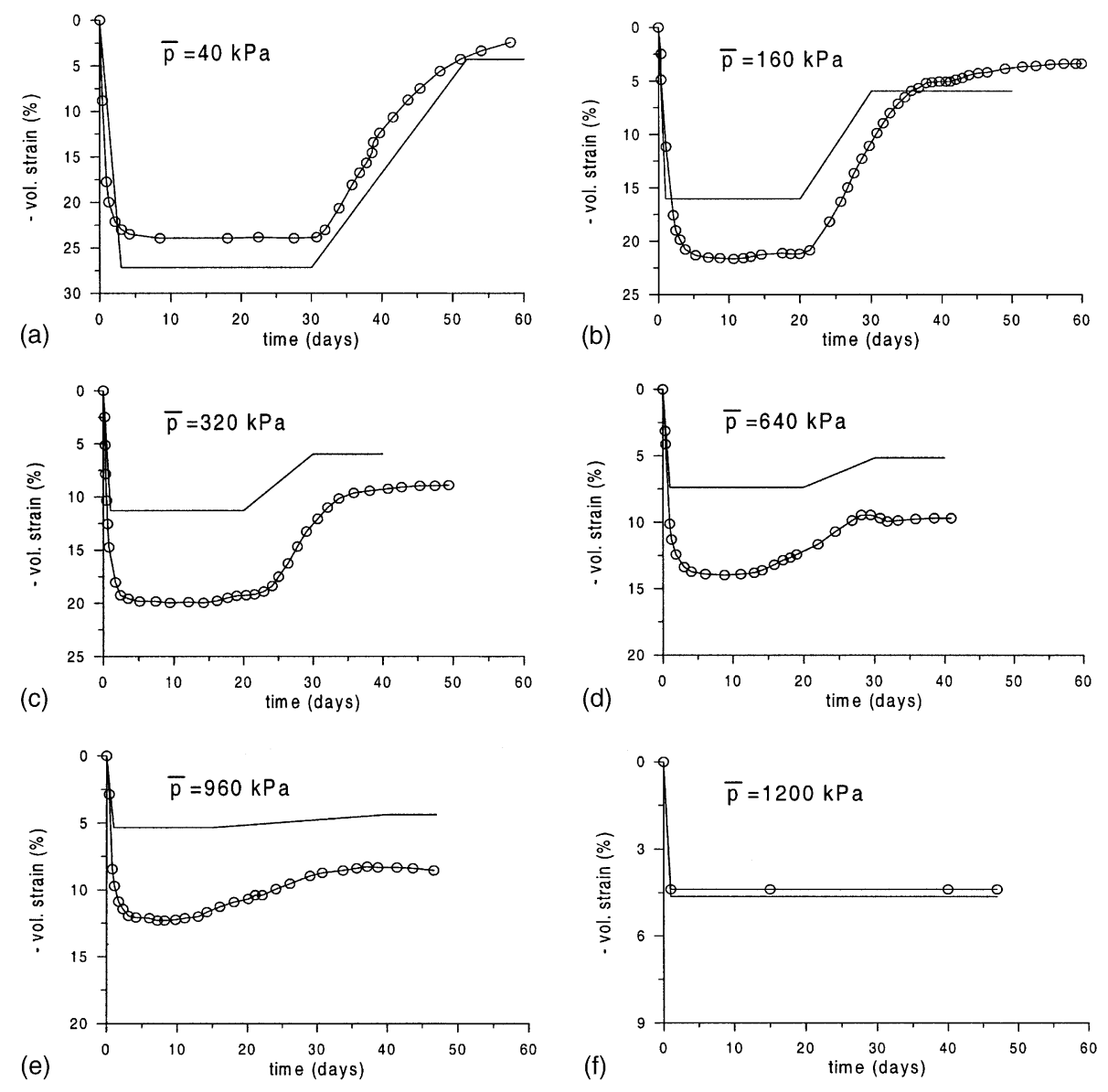

Fig. 7. Chemical cycle at constant effective mean-stress. Model simulations (solid curve) versus experiments by Di Maio (1996, Fig. 7) (symbols). In the model simulations, the timescale is purely fictitious. Increase of salinity of the pore solution by addition of $\mathrm{NaCl}$ up to saturation implies an elastic-plastic consolidation while decrease of salinity occurs elastically, resulting in a net contractancy whose amount increases with the effective mean-stress. The model simulates these features, although the plastic contractancy at large meanstresses is less than the experiment. This discrepancy might be due to the fact that the specimen tested here has not the same properties as the one used to calculate the material parameters, Fig. 6 above: for comparison, both the data and model simulation of the chemical loading at $\bar{p}=1200 \mathrm{kPa}$ from that figure are shown in (f). 
only qualitative. In fact, this apparent discrepancy should be viewed with care, in line with the remarks above concerning the homogeneity of the state of specimens tested and other possible experimental sources of inaccuracy. Indeed, the experimental chemical consolidation (volumetric strain) at $\bar{p}=1200 \mathrm{kPa}$ in Fig. 6 is $0.4 /\left(1+e_{0}\right) \simeq 4.44 \%$ while it is $12 \%$ at $\bar{p}=960 \mathrm{kPa}$ in Fig. 7 . On the other hand, the chemical consolidations from the two figures agree to approximatively $25 \%$ at $\bar{p}=40 \mathrm{kPa}$. Note that the volume changes shown in Fig. 7 of Di Maio (1996) have been calculated with an initial void ratio equal to 13.4 based on information provided by the experimentator. They have been re-scaled by the ratio $(1+13.4) /(1+8)=1.6$ for comparison with her Fig. 8 reproduced in our Fig. 6(a). Since we are concerned here with pointwise material properties, the timescale along the abscissa for simulated results in Fig. 7 is purely fictitious. In order to obtain the time-evolution of the volume change of the sample, a complete initial and boundary value problem including transfer and diffusion effects would have to be considered, Gajo and Loret (2001).

More details of a purely chemical loading are given on Fig. 8 which complements Fig. 7(a) and serves to calibrate the initial slopes $\kappa_{3}$ and $\lambda_{3}$, see Appendices B and D. The slope of the chemical consolidation versus salinity change decreases as the pore water departs from distilled water. On the other hand, increase of salinity above $1 \mathrm{M} \mathrm{NaCl}$ per 1 of solution is practically without effect. Observe that the experimental slope of the chemical consolidation curve is larger than that of the swelling curve, $\lambda_{3}>\kappa_{3}$. Although the experimental loading/unloading curves are not available for Bisaccia clay, the same type of inequality is expected to lead, for that clay, to chemical preconsolidation, Fig. 9.

\subsection{Chemical preconsolidation}

Tests on Bisaccia clay remolded with distilled water have been partially calibrated from the purely mechanical loading cycles shown in Fig. 9 corresponding to distilled and saturated pore solutions. It seems that Bisaccia clay displays a tendency to higher chemical preconsolidation than Ponza bentonite, compare the slopes at the reinitiation of mechanical loading on Figs. 6(e) and 9(b). Even if $\bar{p}_{\kappa}=\bar{p}_{\lambda}$, the model can capture this feature, if $\lambda-\tilde{\kappa}$ is not a constant at varying chemical composition of solid phase as explained in Section 5.1.3. Since $\kappa_{1}=\lambda_{1}$, Table 2, setting $\kappa_{3}=\lambda_{3}$ will not produce preconsolidation, case (a) in Fig. 9. On the other hand, an increasing difference $\lambda_{3}-\kappa_{3}$ implies an increasing preconsolidation, cases (b) and (c) in Fig. 9. Indeed, the variation of $p_{\mathrm{c}}$ due to chemical effects is negative for pore solutions close to distilled water, as can be checked in Eq. (5.12) with the material parameters given in Tables 1 and 2. Hence plasticity occurs right at the beginning of chemical consolidation up to the point where the sign of the derivative of
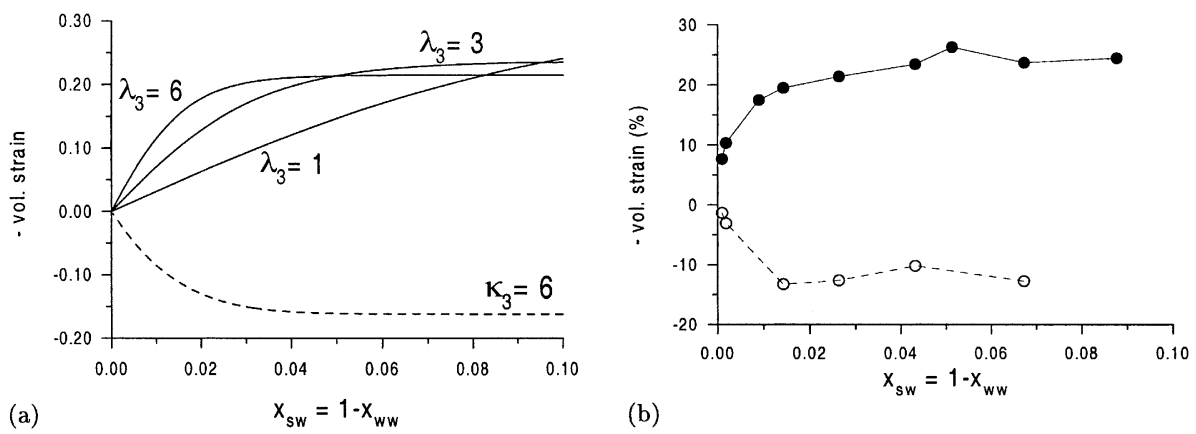

(b)

Fig. 8. Chemical consolidation and swelling of Ponza bentonite starting from distilled water up to $\mathrm{NaCl}$ saturation (solid curve) at constant mechanical load $\bar{p}=80 \mathrm{kPa}$. The essential of the volumetric contractancy occurs experimentally for $\mathrm{NaCl}$ concentrations smaller than $1 \mathrm{~mol} / \mathrm{l}$, that is for molar fractions $x_{\mathrm{sW}}$ of $\mathrm{NaCl}$ inferior to 0.018 . Each of the experimental points corresponds to a single experiment. (a) Model simulations with different initial slopes $\kappa_{3}$, Eqs. (B.1) and (B.2) and ( $\left.\lambda_{3}\right)$, Eqs. (D.1) and (D.2). (b) Experimental data, courtesy Di Maio. 

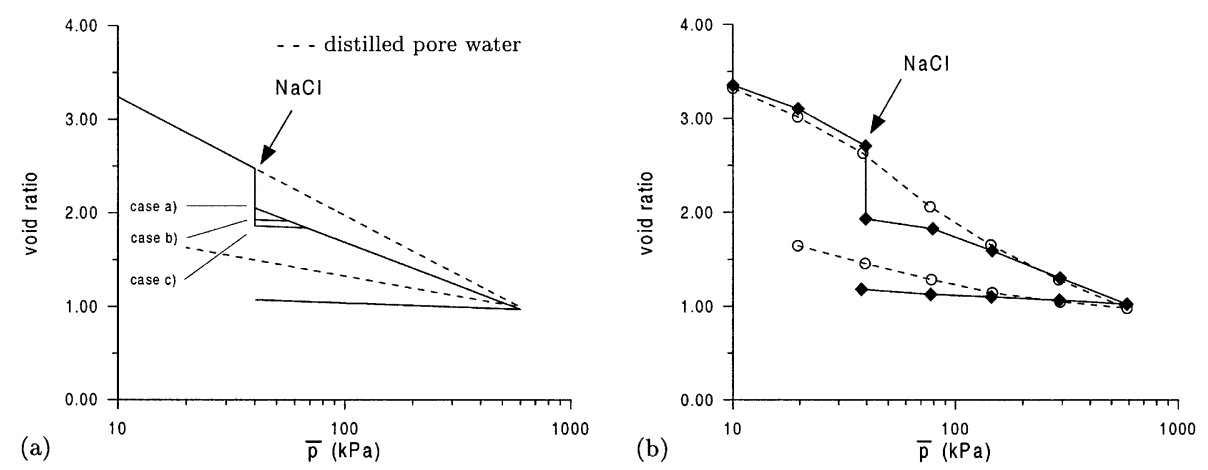

Fig. 9. Mixed chemo-mechanical loading on Bisaccia clay reconstituted with distilled water. The dashed line corresponds to distilled pore water and the solid line to a $\mathrm{NaCl}$ saturated pore water. Experimental data by Di Maio and Fenelli (1997, Fig. 7(a)). For the numerical simulations (a), three cases are shown: (a) $\lambda_{3}=3$; (b) $\lambda_{3}=6$; (c) $\lambda_{3}=9$ while $\kappa_{3}=3$ (see text).
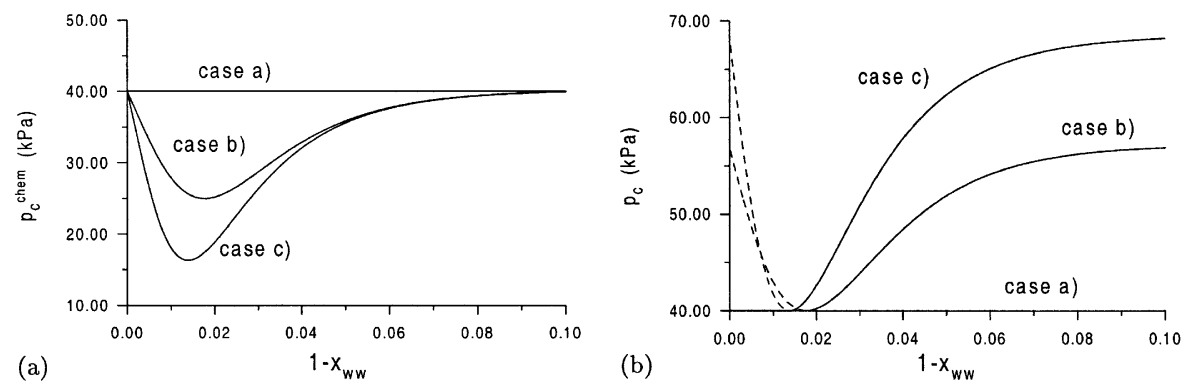

Fig. 10. Evolution of the chemical contribution to the preconsolidation stress $p_{\mathrm{c}}$, (a), and of $p_{\mathrm{c}}$ itself, (b), during chemical consolidation (solid curves) and swelling (dashed curves) for the three cases shown in Fig. 9 at the mean-effective stress $\bar{p}=40 \mathrm{kPa}$. (a) Evolution of the chemical contribution to $p_{\mathrm{c}}$. (b) Evolution of the preconsolidation stress $p_{\mathrm{c}}$.

chemical contribution to $p_{\mathrm{c}}$ changes sign, Fig. 10. There the competition between the negative chemical effect, i.e. softening, and positive plastic effect, i.e. hardening, ceases because the latter is no longer required as the chemical effect becomes positive.

Since here $\bar{p}_{\kappa}=\bar{p}_{\lambda}$, the analytic solution of (5.12) can be easily calculated. In reference to Fig. 5(b), from point $\mathrm{B}$ to point $\mathrm{E}, p_{\mathrm{c}}=\bar{p}$ is constant and (5.12) provides the variation of plastic strain

$$
\operatorname{tr} \epsilon^{\mathrm{pl}}-\operatorname{tr} \epsilon_{\mathrm{B}}^{\mathrm{pl}}=\ln \frac{\bar{p}_{\lambda}}{\bar{p}}\left((\lambda-\tilde{\kappa})-\left(\lambda_{\mathrm{B}}-\tilde{\kappa}_{\mathrm{B}}\right)\right)<0 .
$$

Later on from point $\mathrm{E}$ to $\mathrm{C}$ and $\mathrm{D}$, the behaviour is elastic and (5.12) provides $p_{\mathrm{c}}$,

$$
\frac{p_{\mathrm{c}}}{\bar{p}_{\lambda}}=\left(\frac{\bar{p}}{\bar{p}_{\lambda}}\right)^{(\lambda-\tilde{\kappa}) /\left(\lambda_{\mathrm{E}}-\tilde{\kappa}_{\mathrm{E}}\right)} .
$$

To estimate the chemical contribution to $p_{\mathrm{c}}$, one might assume $p_{\mathrm{c}}=\bar{p}$ in (5.12) (this is an approximation in the elastic range only), and then

$$
{\frac{\Delta p_{\mathrm{c}}}{\bar{p}}}^{\text {chem }}=\ln \frac{\bar{p}_{\lambda}}{\bar{p}} \ln \frac{\lambda-\tilde{\kappa}}{\lambda_{\mathrm{B}}-\tilde{\kappa}_{\mathrm{B}}} .
$$



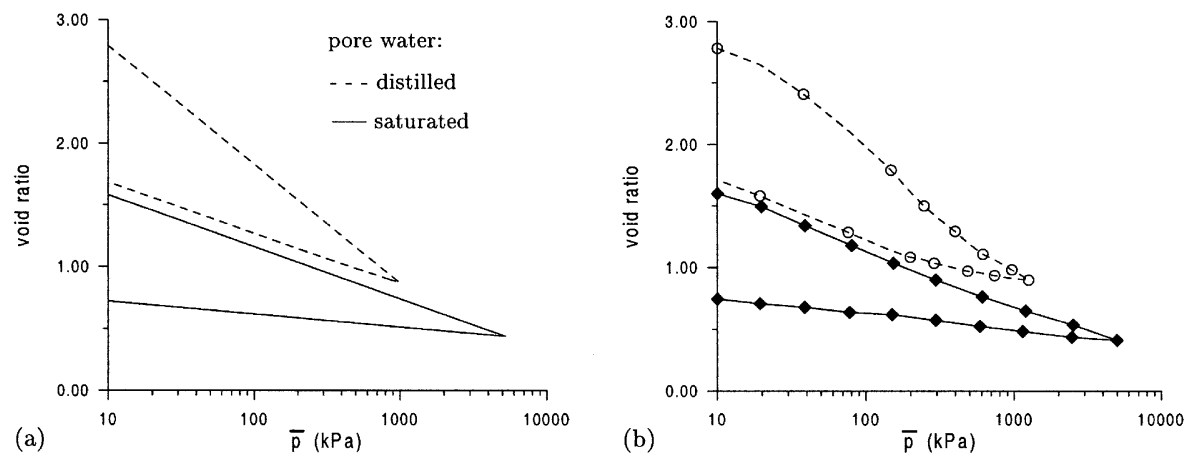

Fig. 11. Purely mechanical load cycle on Bisaccia clay obtained by mixing clay particles with distilled pore water (dashed curve) and a $\mathrm{NaCl}$ saturated solution (solid curve). Experimental data by Di Maio and Onorati (1999, Fig. 4). (a) Model simulations and (b) experimental data.

From Table 1, it turns out that $\lambda^{\text {sat }}-\tilde{\kappa}^{\text {sat }}=\lambda^{\mathrm{dw}}-\tilde{\kappa}^{\mathrm{dw}}$. So, $\delta p_{\mathrm{c}}^{\text {chem }}$ is zero at saturation, point $\mathrm{C}$ in Fig. 5(c), and $p_{\mathrm{c}}$ at point $\mathrm{D}$, where the chemical cycle ends, returns to its value at $\mathrm{C}$. Notice that this special behaviour is closely tied to the values of the parameters in Table 1 mentioned in the lines above.

\subsection{Drained and undrained triaxial tests}

In order to clearly demonstrate that the model is not restricted to a one-dimensional setting and that is embodied in a three-dimensional framework, normally consolidated and overconsolidated undrained triaxial paths performed on a cylindrical apparatus have been simulated, Figs. 12 and 13. Fig. 13 also shows drained triaxial tests. The parameters are partly calibrated from the data corresponding to mechanical loadings on Bisaccia clay remolded with a saline pore solution, Fig. 11. These tests indicate a very strong influence of the pore water chemical composition on the friction angle $\varphi$, i.e. in our model on the generalized Cam-Clay coefficient $M$ equal for triaxial compression to $6 \sin \varphi /(3-\sin \varphi)$ : a saline pore water implies water desorption and thus increases the value of the friction angle, as already noted by Mesri and Olson (1970).

\section{Concluding remarks}

The model developed in this paper is embedded in a general three-dimensional framework that can be viewed as extending the classic elastic-plastic framework for porous media to account for chemomechanical couplings. Therefore, it can capitalize upon the available theoretical and computational developments in view of solving initial and boundary value problems in a multi-dimensional setting. The local interphase mass transfer phenomena have to be accounted for in addition to diffusion. Although analyses of instrumented in situ cases are the ultimate goal, it is necessary in a first step to simulate laboratory tests to check the validity of certain assumptions that have been made to interpret the experimental results, e.g. assumption of chemical equilibrium and uniformity of the chemical fields throughout the sample at the times where measurements are made. Such a companion analysis is performed in Gajo and Loret (2001).

An extension of the framework is necessary to analyze natural clays where several cations co-exist. The electrolytic nature of the solutions requires to account for electro-neutrality and replace chemical potentials by electro-chemical potentials. This subject is examined in Gajo et al. (2002), with finite element analysis of boundary value problems in Gajo and Loret (2002). On the other hand, the present formulation is thought 


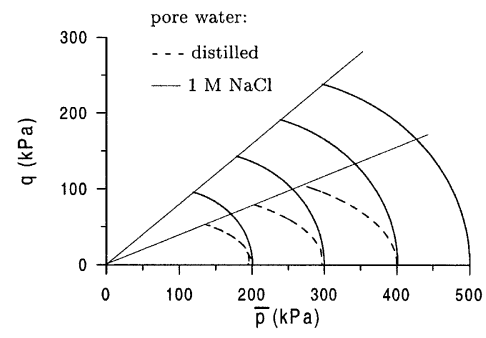

(a) Model simulations

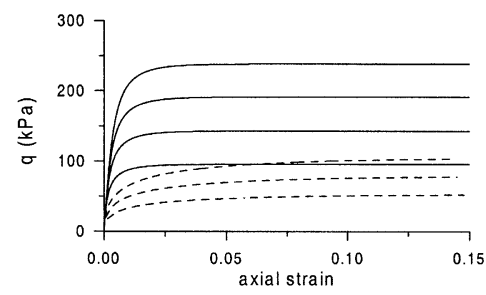

(c) Model simulations.

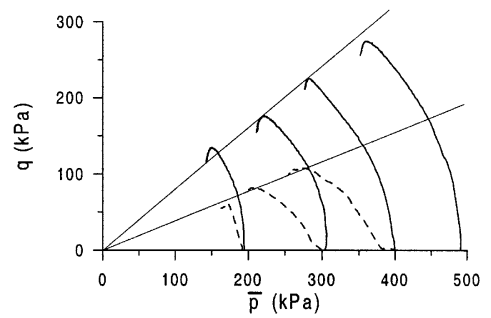

(b) Experimental data.

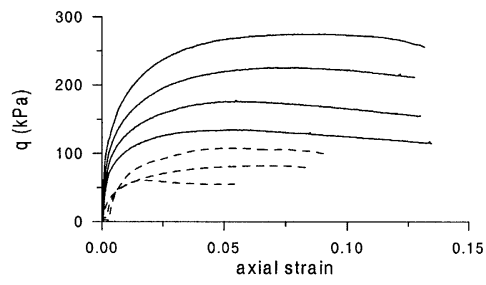

(d) Experimental data.

Fig. 12. Undrained tests with different initial effective mean-stresses on normally consolidated Bisaccia clay obtained by mixing clay particles with distilled water (dashed curves) and with a 1 molar $\mathrm{NaCl}$ solution (solid curves). The specimen mixed with a saline pore water has an increased strength (friction angle $\varphi^{\text {sat }}=20.7^{\circ}$ ) with respect to the specimen with distilled pore water (friction angle $\varphi^{\mathrm{dw}}=10.8^{\circ}$ ). Experimental data (b) by Di Maio and Onorati (1999, Fig. 9), and (d) courtesy Di Maio. (a) Model simulations, (b) experimental data, (c) model simulations and (d) experimental data.
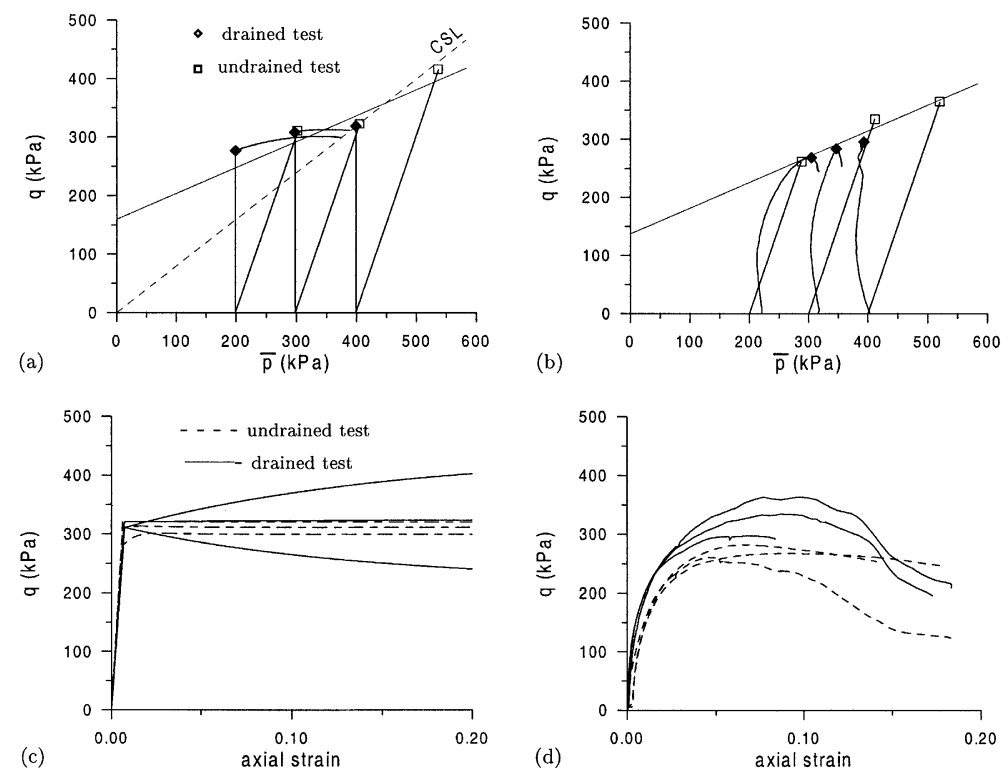

Fig. 13. Drained and undrained triaxial tests with different initial effective mean-stresses on Bisaccia clay overconsolidated to $800 \mathrm{kPa}$ and obtained by mixing clay particles with a $1 \mathrm{M} \mathrm{NaCl}$ solution. The friction angle is $\varphi^{\mathrm{sat}}=20.7^{\circ}$ as in the previous plot. Experimental data (b) by Di Maio and Onorati (1999, Fig. 11), and (d) courtesy Di Maio. (a) Model simulations, (b) experimental data, (c) model simulations and (d) experimental data. 
to constitute at least a useful tentative framework to analyze the behaviour of clays submitted to non-ionic solutes, such as organic pollutants.

Implicit in this work is the assumption that the experiments used are isothermal. However, we have no information on this aspect: it would be interesting to have available chemo-mechanical experiments with controlled fixed temperature, and further with controlled varying temperature in order to assess the importance of the thermo-chemo-mechanical couplings.

Finally, to the authors's surprise, no consistent database exists analogous to that of experiments by Di Maio and coworkers referred here, regarding processes that begin with a decrease of ionic concentration of pore water.

\section{Acknowledgements}

The authors thank C. Di Maio, Università della Basilicata, for providing them with several unpublished experimental data and for help in their interpretation. TH acknowledges a senior fellowship from the french Ministère de l'Enseignement Supérieur et de la Recherche and AG a visiting fellowship at Ecole Nationale Supérieure d'Hydraulique et Mécanique de Grenoble.

\section{Appendix A. Mass balance equations for incompressible species}

For the species $k$ of the phase $K$, the balance of mass reads, e.g. Bowen (1976),

$$
\frac{\mathrm{d}^{k K}}{\mathrm{~d} t} \rho^{k K}+\rho^{k K} \operatorname{div} \mathbf{v}_{k K}=\hat{\rho}^{k K}
$$

where the symbol $\mathrm{d}^{k K} / \mathrm{d} t$ denotes the derivative following the particles of species $k$ of phase $K .{ }^{5}$ It can be expressed in terms of the derivative following solid particles,

$$
\frac{\mathrm{d}^{k K}}{\mathrm{~d} t}(\cdot)=\frac{\mathrm{d}^{\mathrm{S}}}{\mathrm{d} t}(\cdot)+\nabla(\cdot) \cdot\left(\mathbf{v}_{k K}-\mathbf{v}_{\mathrm{S}}\right) .
$$

Using the definition (2.15) and the incompressibility condition, the above balance of mass can be recast in terms of the volume fraction $n_{k K}$,

$$
\frac{\mathrm{d}^{k K}}{\mathrm{~d} t} n_{k K}+n_{k K} \operatorname{div} \mathbf{v}_{k K}=\frac{\hat{\rho}^{k K}}{\rho_{k K}} .
$$

Summing up for the species of each phase and using the volume contents (2.13), one obtains the balance of mass for phase $K$ as,

$$
\frac{\mathrm{d}^{\mathrm{S}} v_{K}}{\mathrm{~d} t}+\operatorname{div}\left(\sum_{k \in K} n_{k K} \mathbf{v}_{k K}-n_{K} \mathbf{v}_{\mathrm{S}}\right)=\sum_{k \in K} \frac{\hat{\rho}^{k K}}{\rho_{k K}} .
$$

Therefore, we have for the mass contents of the phases (2.14),

\footnotetext{
${ }^{5}$ In the text, the derivative $\mathrm{d}^{\mathrm{S}} / \mathrm{d} t$ is denoted by $\delta / \delta t$.
} 


$$
\begin{aligned}
\frac{\mathrm{d}^{\mathrm{S}} m_{\mathrm{S}}}{\mathrm{d} t} & =\sum_{k \in \mathrm{S}} \hat{\rho}^{k \mathrm{~S}}, \\
\frac{\mathrm{d}^{\mathrm{S}} m_{\mathrm{W}}}{\mathrm{d} t}= & \overbrace{\text { reactive part }}^{\sum_{k \in \mathrm{W}} \hat{\rho}^{k \mathrm{~W}}-\operatorname{div}\left(\sum_{\mathrm{k} \in \mathrm{W}} \mathbf{M}_{\mathrm{kW}}\right)}
\end{aligned}
$$

with $\mathbf{M}_{k \mathrm{~W}}=\rho^{k \mathrm{~W}}\left(\mathbf{v}_{k \mathrm{~W}}-\mathbf{v}_{\mathrm{S}}\right)$ flux defined by (3.3). These relations correspond to our assumptions that the species (salt and absorbed water) of the solid phase are only reactive,

$$
\frac{\mathrm{d}^{\mathrm{S}} m_{k \mathrm{~S}}}{\mathrm{~d} t}=\hat{\rho}^{k \mathrm{~S}}, \quad k \in \mathrm{S},
$$

while the species of the fluid phase are both diffusive and reactive, that is, mass changes are due both to transfer into/from the solid phase and to exchange with the exterior of the representative elementary volume.

The incompressibility condition may be cast in the formula, obtained by summing (A.4) for $K=\mathrm{S}$ and $K=\mathrm{W}$,

$$
\operatorname{div}\left(n_{\mathrm{S}} \mathbf{v}_{\mathrm{S}}+\sum_{k \in \mathrm{W}} n_{k \mathrm{~W}} \mathbf{v}_{k \mathrm{~W}}\right)=\sum_{k, K} \frac{\hat{\rho}^{k K}}{\rho_{k K}},
$$

hence

$$
\frac{\mathrm{d}^{\mathrm{S}} v_{\mathrm{W}}}{\mathrm{d} t}=\operatorname{div} \mathbf{v}_{\mathrm{S}}-\sum_{k \in \mathrm{S}} \frac{\hat{\rho}^{k \mathrm{~S}}}{\rho_{k \mathrm{~S}}}=\operatorname{div} \mathbf{v}_{\mathrm{S}}-\sum_{k \in \mathrm{S}} \frac{1}{\rho_{k \mathrm{~S}}} \frac{\mathrm{d}^{\mathrm{S}} m_{k \mathrm{~S}}}{\mathrm{~d} t} .
$$

\section{Appendix B. Chemical interpolation for the elastic coefficient $\boldsymbol{\kappa}=\boldsymbol{\kappa}\left(\boldsymbol{m}_{\mathrm{wS}}^{\mathrm{el}}\right)$}

To estimate the effect of pore water composition on the elastic coefficient $\kappa$, one has in a first step to find the associated mass of absorbed water at reference states. Now, the elastic coefficient $\kappa$ depends on the mass of reversibly absorbed water $m_{\mathrm{wS}}^{\mathrm{el}}$, or equivalently, since only the mass of water is changing in the solid phase, on the molar fraction $x_{\mathrm{wS}}^{\mathrm{el}}$ : the latter is obtained from the elastic mass of absorbed water and the masses of the other species through the definition (2.16). Although an abuse of notation, we note $\kappa\left(x_{\mathrm{wS}}^{\mathrm{el}}\right)=\kappa\left(m_{\mathrm{wS}}^{\mathrm{el}}\right)$. For example, we may measure the values of $\kappa^{\mathrm{dw}}=\kappa\left(x_{\mathrm{wS}}^{\mathrm{el}, \mathrm{dw}}\right)$ and $\kappa^{\mathrm{sat}}=\kappa\left(x_{\mathrm{wS}}^{\mathrm{el}, \mathrm{sat}}\right)$ corresponding, respectively, to distilled and salt-saturated pore water.

Then the chemical effect on $\kappa$ is introduced through interpolation between these two extreme situations, for example

$$
\kappa\left(x_{\mathrm{wS}}^{\mathrm{el}}\right)=\kappa_{1} \Phi\left(x_{\mathrm{wS}}^{\mathrm{el}}\right)+\kappa_{2},
$$

where the $\kappa_{i}$ 's, $i=1,2$, are constants. The hyperbolic tangent is chosen as interpolation function $\Phi$,

$$
\Phi\left(x_{\mathrm{wS}}^{\mathrm{el}}\right)=\tanh \left(\kappa_{3} \frac{x_{\mathrm{wS}}^{\mathrm{el}}-x_{\mathrm{wS}}^{\mathrm{el}, \mathrm{dw}}}{x_{\mathrm{wS}}^{\mathrm{el}, \mathrm{sat}}-x_{\mathrm{wS}}^{\mathrm{el}, \mathrm{dw}}}\right) .
$$

The variation of pore water composition is observed to lead to fast changes of mechanical properties especially at small departure from distilled water. So, the constant $\kappa_{3}$ can be obtained from the slope $\mathrm{d} \kappa / \mathrm{d} x_{\mathrm{wS}}^{\mathrm{el}}$ at $x_{\mathrm{wS}}^{\mathrm{el}}=x_{\mathrm{wS}}^{\mathrm{el}, \mathrm{dw}}$, namely 


$$
\frac{\kappa_{3}}{\tanh \left(\kappa_{3}\right)}=\frac{x_{\mathrm{wS}}^{\mathrm{el}, \mathrm{sat}}-x_{\mathrm{wS}}^{\mathrm{el}, \mathrm{dw}}}{\kappa^{\mathrm{sat}}-\kappa^{\mathrm{dw}}} \frac{\mathrm{d} \kappa}{\mathrm{d} x_{\mathrm{wS}}^{\mathrm{el}}}\left(x_{\mathrm{wS}}^{\mathrm{el}}=x_{\mathrm{wS}}^{\mathrm{el,dw}}\right), \quad \kappa_{1}=\frac{\kappa^{\mathrm{sat}}-\kappa^{\mathrm{dw}}}{\tanh \left(\kappa_{3}\right)}, \quad \kappa_{2}=\kappa^{\mathrm{dw}} .
$$

The following derivatives are needed,

$$
\frac{\mathrm{d} \kappa}{\mathrm{d} m_{\mathrm{wS}}^{\mathrm{el}}}=\frac{\mathrm{d} \kappa}{\mathrm{d} x_{\mathrm{wS}}^{\mathrm{el}}} \frac{\mathrm{d} x_{\mathrm{wS}}^{\mathrm{el}}}{\mathrm{d} m_{\mathrm{wS}}^{\mathrm{el}}}, \quad \frac{\mathrm{d}^{2} \kappa}{\mathrm{d}^{2} m_{\mathrm{wS}}^{\mathrm{el}}}=\frac{\mathrm{d}^{2} \kappa}{\mathrm{d}^{2} x_{\mathrm{wS}}^{\mathrm{el}}}\left(\frac{\mathrm{d} x_{\mathrm{wS}}^{\mathrm{el}}}{\mathrm{d} m_{\mathrm{wS}}^{\mathrm{el}}}\right)^{2}-2 \frac{x_{\mathrm{wS}}^{\mathrm{el}}}{m_{\mathrm{wS}}^{\mathrm{el}}} \frac{\mathrm{d} \kappa}{\mathrm{d} x_{\mathrm{wS}}^{\mathrm{el}}} \frac{\mathrm{d} x_{\mathrm{wS}}^{\mathrm{el}}}{\mathrm{d} m_{\mathrm{wS}}^{\mathrm{el}}} .
$$

They make use of the general relation (2.17) applied to elastic entities and of the specific formulas deduced from (B.1),

$$
\frac{\mathrm{d} \kappa}{\mathrm{d} x_{\mathrm{wS}}^{\mathrm{el}}}=\kappa_{1} \kappa_{3} \frac{1-\Phi^{2}\left(x_{\mathrm{wS}}^{\mathrm{el}}\right)}{x_{\mathrm{wS}}^{\mathrm{el}, \mathrm{sat}}-x_{\mathrm{wS}}^{\mathrm{el}, \mathrm{dw}}}, \quad \frac{\mathrm{d}^{2} \kappa}{\mathrm{d}^{2} x_{\mathrm{wS}}^{\mathrm{el}}}=-2 \kappa_{1} \kappa_{3}^{2} \Phi\left(x_{\mathrm{wS}}^{\mathrm{el}}\right) \frac{1-\Phi^{2}\left(x_{\mathrm{wS}}^{\mathrm{el}}\right)}{\left(x_{\mathrm{wS}}^{\mathrm{el}, \mathrm{sat}}-x_{\mathrm{wS}}^{\mathrm{el}, \mathrm{dw}}\right)^{2}} .
$$

\section{Appendix C. Elastic-plastic stiffness}

The coefficients $f_{p}, f_{q}, f_{\mathrm{w}}, g_{p}, g_{q}, g_{\mathrm{w}}$ entering the elastic-plastic stiffness (5.7) are obtained from the consistency condition $\delta f=0$ :

$$
\begin{aligned}
& f_{p}=-B \frac{\partial f}{\partial \bar{p}}+B_{\mathrm{w}} \frac{\partial f}{\partial \bar{\mu}_{\mathrm{wS}}}, \quad g_{p}=-B \frac{\partial g}{\partial \bar{p}}+B_{\mathrm{w}} \frac{\partial g}{\partial \bar{\mu}_{\mathrm{wS}}}, \\
& f_{q}=3 G \frac{\partial f}{\partial q}, \quad g_{q}=3 G \frac{\partial g}{\partial q}, \\
& f_{\mathrm{w}}=-B_{\mathrm{w}} \frac{\partial f}{\partial \bar{p}}+\beta \frac{\partial f}{\partial \bar{\mu}_{\mathrm{wS}}}, \quad g_{\mathrm{w}}=-B_{\mathrm{w}} \frac{\partial g}{\partial \bar{p}}+\beta \frac{\partial g}{\partial \bar{\mu}_{\mathrm{wS}}} .
\end{aligned}
$$

The plastic modulus $H$ is assumed to take only strictly positive values,

$$
H=h+B \frac{\partial f}{\partial \bar{p}} \frac{\partial g}{\partial \bar{p}}+3 G \frac{\partial f}{\partial q} \frac{\partial g}{\partial q}-B_{\mathrm{w}}\left(\frac{\partial f}{\partial \bar{p}} \frac{\partial g}{\partial \bar{\mu}_{\mathrm{wS}}}+\frac{\partial g}{\partial \bar{p}} \frac{\partial f}{\partial \bar{\mu}_{\mathrm{wS}}}\right)+\beta \frac{\partial f}{\partial \bar{\mu}_{\mathrm{wS}}} \frac{\partial g}{\partial \bar{\mu}_{\mathrm{wS}}}
$$

while the hardening modulus $h$ will be positive for hardening, and negative for softening,

$$
h=\frac{p_{\mathrm{c}}}{\lambda-\tilde{\kappa}} \frac{\partial g}{\partial \bar{p}} .
$$

For the Modified Cam-Clay model (5.8), the following derivatives are needed,

$$
\frac{\partial f}{\partial \bar{p}}=1-\frac{1}{M^{2}} \frac{q^{2}}{\bar{p}^{2}}, \quad \frac{\partial f}{\partial q}=\frac{2}{M^{2}} \frac{q}{\bar{p}}, \quad \frac{\partial f}{\partial \bar{\mu}_{\mathrm{wS}}}=-\frac{2}{M^{3}} \frac{q^{2}}{\bar{p}} \frac{\mathrm{d} M}{\mathrm{~d} \bar{\mu}_{\mathrm{wS}}}-\frac{\partial p_{\mathrm{c}}}{\partial \bar{\mu}_{\mathrm{wS}}},
$$

where

$$
\frac{\partial p_{\mathrm{c}}}{\partial \bar{\mu}_{\mathrm{wS}}}=\left(\ln \frac{\bar{p}_{\lambda}}{p_{\mathrm{c}}} \frac{\partial \lambda}{\partial \bar{\mu}_{\mathrm{wS}}}-\ln \frac{\bar{p}_{\kappa}}{p_{\mathrm{c}}} \frac{\partial \tilde{\kappa}}{\partial \bar{\mu}_{\mathrm{wS}}}\right) \frac{p_{\mathrm{c}}}{\lambda-\tilde{\kappa}} .
$$

\section{Appendix D. Chemical interpolation for the plastic coefficients $\lambda$ and $M$}

To estimate the effect of pore water composition on the plastic coefficients $\lambda$ and $M$, one has in a first step to find the associated chemical potential of absorbed water. At equilibrium, the latter is simply equal to the 
chemical potential of pore water. ${ }^{6}$ Since the chemical effects on these two coefficients are assumed to be similar, we just consider one of them, say $\lambda=\lambda\left(\bar{\mu}_{\mathrm{wS}}\right)$.

We may measure the values of $\lambda^{\mathrm{dw}}=\lambda\left(\bar{\mu}_{\mathrm{wS}}^{\mathrm{dw}}\right)$ and $\lambda^{\mathrm{sat}}=\lambda\left(\bar{\mu}_{\mathrm{wS}}^{\mathrm{sat}}\right)$ corresponding, respectively, to distilled and salt-saturated pore water. Then the chemical effect on $\lambda$ is introduced through interpolation between these two extreme situations, for example

$$
\lambda\left(\bar{\mu}_{\mathrm{wS}}\right)=\lambda_{1} \Phi\left(\bar{\mu}_{\mathrm{wS}}\right)+\lambda_{2},
$$

where the $\lambda_{i}$ 's, $i=1,2$, are constants. The hyperbolic tangent is taken as the interpolation function,

$$
\Phi\left(\bar{\mu}_{\mathrm{wS}}\right)=\tanh \left(\lambda_{3} \frac{\phi\left(\bar{\mu}_{\mathrm{wS}}\right)-\phi\left(\bar{\mu}_{\mathrm{wS}}^{\mathrm{dw}}\right)}{\phi\left(\bar{\mu}_{\mathrm{wS}}^{\mathrm{sat}}\right)-\phi\left(\bar{\mu}_{\mathrm{wS}}^{\mathrm{dw}}\right)}\right),
$$

where

$$
\phi\left(\bar{\mu}_{\mathrm{wS}}\right)=\exp \left(\frac{m_{\mathrm{w}}^{(\mathrm{M})}}{R T} \bar{\mu}_{\mathrm{wS}}\right) .
$$

The constant $\lambda_{3}$ can be obtained from the slope $\mathrm{d} \lambda / \mathrm{d} \bar{\mu}_{\mathrm{wS}}$ at $\bar{\mu}_{\mathrm{wS}}=\bar{\mu}_{\mathrm{wS}}^{\mathrm{dw}}$

$$
\frac{\lambda_{3}}{\tanh \left(\lambda_{3}\right)}=\frac{\phi\left(\bar{\mu}_{\mathrm{wS}}^{\mathrm{sat}}\right)-\phi\left(\bar{\mu}_{\mathrm{wS}}^{\mathrm{dw}}\right)}{\lambda^{\mathrm{sat}}-\lambda^{\mathrm{dw}}} \frac{\mathrm{d} \lambda}{\mathrm{d} \bar{\mu}_{\mathrm{wS}}}\left(\frac{\mathrm{d} \phi}{\mathrm{d} \bar{\mu}_{\mathrm{wS}}}\right)^{-1}\left(\bar{\mu}_{\mathrm{wS}}=\bar{\mu}_{\mathrm{wS}}^{\mathrm{dw}}\right), \quad \lambda_{1}=\frac{\lambda^{\mathrm{sat}}-\lambda^{\mathrm{dw}}}{\tanh \left(\lambda_{3}\right)}, \quad \lambda_{2}=\lambda^{\mathrm{dw}} .
$$

The following derivatives are needed,

$$
\frac{\mathrm{d} \phi}{\mathrm{d} \bar{\mu}_{\mathrm{wS}}}=\frac{m_{\mathrm{w}}^{(\mathrm{M})}}{R T} \phi\left(\bar{\mu}_{\mathrm{wS}}\right), \quad \frac{\mathrm{d} \lambda}{\mathrm{d} \bar{\mu}_{\mathrm{wS}}}=\lambda_{1} \lambda_{3} \frac{1-\Phi^{2}\left(\bar{\mu}_{\mathrm{wS}}\right)}{\phi\left(\bar{\mu}_{\mathrm{wS}}^{\mathrm{sat}}\right)-\phi\left(\bar{\mu}_{\mathrm{wS}}^{\mathrm{dw}}\right)} \frac{\mathrm{d} \phi}{\mathrm{d} \bar{\mu}_{\mathrm{wS}}} .
$$

\section{Appendix E. Determination of the initial state in the solid phase}

We seek for an approximation of the state in the solid phase while the chemical composition of pore water corresponds to distilled water. Thus $x_{\mathrm{wW}} \sim 1$ is large and $x_{\mathrm{sW}}=1-x_{\mathrm{wW}} \sim 0$. To ensure electroneutrality, cations should be present in the solid phase to counterbalance the negatively charged clay particles. Since we consider a single salt in this analysis, to maintain electroneutrality in both phases, cations $\mathrm{Na}^{+}$and anions $\mathrm{Cl}^{-}$need to transfer together. Let us concentrate on the case where the membrane is permeable to water only. Since we are considering a situation in which pore water is distilled, we take the rough approximation to agglomerate salt and clay in a single species in the determination of initial conditions. In fact, for compacted clay, Ransom and Helgeson (1994) find 15 moles of $\mathrm{H}_{2} \mathrm{O}$ and 3 moles of clay per mole of monovalent cation. So we set formally $N_{\mathrm{s} S}=0$. Therefore, the chemical composition of the solid phase requires just $x_{\mathrm{wS}}$. As shown below, its determination in turn requires the knowledge of (a) the initial void ratio $e$ and (b) some guess on the ratio of volumes of absorbed water and free water.

For that purpose, the void ratio $e$ is roughly approximated as $\left(V_{\mathrm{wW}}+V_{\mathrm{wS}}\right) / V_{\mathrm{S}}$ as if absorbed water could be entirely removed through heating at $105^{\circ}$. A more accurate approximation is presented in Gajo et al. (2002). Assuming the molar volumes of pore and absorbed water to be identical, namely $v_{\mathrm{wW}}^{(\mathrm{M})}=v_{\mathrm{wS}}^{(\mathrm{M})}=$ $18 \mathrm{~cm}^{3}$, and using the fact that the volume occupied by any species is equal to the number of moles times its molar volume, the void ratio becomes,

\footnotetext{
${ }^{6}$ In fact, the equality holds to within an unknown constant. So in practice, initially one has first to find the molar fraction $x_{\mathrm{wS}}^{\mathrm{el}}$ and next one approximates $\bar{\mu}_{\mathrm{wS}}$ by $R T / m_{\mathrm{w}}^{(\mathrm{M})} \ln x_{\mathrm{wS}}^{\mathrm{el}}$.
} 


$$
e=\frac{N_{\mathrm{wS}}}{N_{\mathrm{cS}}}\left(1+\frac{N_{\mathrm{wW}}}{N_{\mathrm{wS}}}\right) \frac{v_{\mathrm{wS}}^{(\mathrm{M})}}{v_{\mathrm{cS}}^{(\mathrm{M})}} .
$$

Under the assumption that the number of moles of salt in the solid phase is small with respect to that of clay and absorbed water, the molar fraction of absorbed water $x_{\mathrm{wS}}=N_{\mathrm{wS}} /\left(N_{\mathrm{wS}}+N_{\mathrm{cS}}\right)$ is obtained from (E.1) as

$$
x_{\mathrm{wS}} \sim \frac{1}{1+\left(N_{\mathrm{cS}} / N_{\mathrm{wS}}\right)}=1 /\left[1+\frac{1}{e}\left(1+\frac{N_{\mathrm{wW}}}{N_{\mathrm{wS}}}\right) \frac{v_{\mathrm{wS}}^{(\mathrm{M})}}{v_{\mathrm{cS}}^{(\mathrm{M})}}\right] .
$$

Hence, determination of the initial molar fractions $x_{\mathrm{wS}}$ and $x_{\mathrm{cS}}$ requires the knowledge of the (easily accessible) initial void ratio $e$ and some guess on the ratio of volumes of absorbed water and free water.

In a unit volume of porous medium, the number of moles of absorbed water is, using (E.1),

$$
N_{\mathrm{wS}} \sim \frac{e}{1+e} \frac{1}{1+\left(N_{\mathrm{wW}} / N_{\mathrm{wS}}\right)} \frac{1}{v_{\mathrm{wS}}^{(\mathrm{M})}}
$$

from which the volume and mass of absorbed water per unit volume of porous medium can be estimated, namely $v_{\mathrm{wS}}=N_{\mathrm{wS}} v_{\mathrm{wS}}^{(\mathrm{M})}$ and $m_{\mathrm{wS}}=N_{\mathrm{wS}} m_{\mathrm{w}}^{(\mathrm{M})}$, with $m_{\mathrm{w}}^{(\mathrm{M})}=18 \mathrm{~g}$. The volume and mass of free water are equal to their counterparts in the solid phase times $N_{\mathrm{wW}} / N_{\mathrm{wS}}$.

The number of moles of clay per unit volume of porous medium is,

$$
N_{\mathrm{cS}}=\frac{1}{1+e} \frac{1}{v_{\mathrm{cS}}^{(\mathrm{M})}},
$$

from which the volume and mass of the clay particles are $v_{\mathrm{cS}}=N_{\mathrm{cS}} v_{\mathrm{cS}}^{(\mathrm{M})}$ and $m_{\mathrm{cS}}=N_{\mathrm{cS}} m_{\mathrm{c}}^{(\mathrm{M})}$. Using the technique of structural analogy, Ransom and Helgeson (1994) have obtained $v_{\mathrm{cS}}^{(\mathrm{M})} \sim 146 \mathrm{~cm}^{3}$ for an anhydrous smectite of formula $\mathrm{NaAl}_{3} \mathrm{Si}_{3} \mathrm{O}_{10}(\mathrm{OH})_{2}$ of molar mass $m_{\mathrm{c}}^{(\mathrm{M})}=382 \mathrm{~g}$.

Once the initial condition corresponding to distilled pore water has been obtained, the state of the solid phase in equilibrium with pore water saturated by salt is deduced simply by solving the equation(s) of equilibrium of the chemical potential(s) of the species that can cross the membrane.

\section{References}

Anandarajah, R., Lu, N., 1991. Numerical study of the electrical double layer repulsion between non-parallel clay particles of finite length. Int. J. Numer. Anal. Meth. Geomech. 55, 683-702.

Bataille, J., Kestin, J., 1977. Thermodynamics of mixtures. J. Non-Equil. Thermody. 2, 49-65.

Bennethum, L.S., Cushman, J.H., 1999. Coupled solvent and heat transport of a mixture of swelling porous particles and fluids: single time-scale problem. Transport Porous Media. 36, 211-244.

Boek, E.S., Convey, P.V., Skipper, N.T., 1995. Molecular modeling of clay hydration: a study of hysteresis loops in the swelling curves of sodium montmorillonite. Langmuir 11, 4629-4631.

Bolt, G.H., 1956. Physico-chemical analysis of the compressibility of pure clays. Géotechnique 6 (2), 86-93.

Bowen, R.M., 1976. Theory of mixtures. In: Eringen, A.C. (Ed.), Continuum Physics, vol. 3. Academic Press, New York, pp. 1-127.

Charlez, Ph., Pradet, V., Pollard, R., Onaisi A., Grégoire, M., 1998. How to manage wellbore stability in the Vicking Graben tertiary shales by using mud systems environmentally friendly? Offshore Technology Conference, Houston, 4-7 May, 1998.

Delville, A., 1991. Modeling the clay-water interface. Langmuir 7, 547-555.

Delville, A., 1992. Structure of liquids at a solid interface: application to the swelling of clay by water. Langmuir 8, $1796-1805$.

Di Maio, C., 1996. Exposure of bentonite to salt solution: osmotic and mechanical effects. Géotechnique 46 (4), 695-707.

Di Maio, C., Fenelli, G., 1997. Influenza delle interazioni chimico-fisiche sulla deformabilità di alcuni terreni argillosi. Rivista Italiana di Geotecnica 1, 695-707.

Di Maio, C., Onorati, R., 1999. Prove di laboratorio: influenza di composizione del liquido di cella. In: Rendiconti del XX Convegno Nazionale di Geotecnica, Parma, pp. 87-94.

Eringen, A.C., Ingram, J.D., 1965. A continuum theory for chemically reacting media-I. Int. J. Eng. Sci. 3, $197-212$. 
Fu, M.H., Zhang, Z.Z., Low, P.F., 1990. Changes in the properties of montmorillonite-water system during the adsorption and desorption of water. Clays and Clay Minerals 38, 485-492.

Gajo, A., Loret, B., 2001. Finite element simulations of chemo-mechanical coupling in elastic-plastic homoionic expansive clays. submitted.

Gajo, A., Loret, B., 2002. Finite element simulations of electro-chemo-mechanical couplings in elastic-plastic heteroionic expansive clays. submitted.

Gajo, A., Loret, B., Hueckel, T., 2002. Electro-chemo-mechanical couplings in saturated porous media: elastic-plastic behaviour of heteroionic expansive clays. Int. J. Solids Structures, submitted.

Haase, R., 1990. Thermodynamics of Irreversible Processes. Dover Publications, New York.

Heidug, W.K., Wong, S.-W., 1996. Hydration swelling of water-absorbing rocks: a constitutive model. Int. J. Numer. Anal. Meth. Geomech. 20, 402-430.

Huang, W.L., Bassett, W.A., Wu, T.C., 1994. Dehydration and rehydration of montmorillonite at elevated temperatures monitored using synchrotron radiation. Am. Mineral. 79, 683-691.

Hueckel, T., 1992a. Water-mineral interaction in hygro-mechanics of clays exposed to environmental loads: a mixture approach. Can. Geotech. J. 29, 1071-1086.

Hueckel, T., 1992b. On effective stress concepts and deformation in clays subjected to environmental loads. Can. Geotech. J. 29, 11201125.

Huyghe, J.M., Janssen, J.D., 1999. Thermo-chemo-electro-mechanical formulation of saturated charged porous solids. Transport Porous Media. 34 (1-3), 129-141.

Israelachvili, J., 1992. Intermolecular and surface forces. Academic Press, London, UK.

Karaborni, S., Smit, B., Heidug, W., Urai, J., van Oort, E., 1996. The swelling of clays: molecular simulations of the hydration of montmorillonite. Science 271, 1102-1105.

Karalis, T.K., 1993. Water flow in non-saturated swelling soil. Int. J. Eng. Sci. 31 (5), 751-774.

Kestin, J., 1968. A Course in Thermodynamics. Blaisdell Publishing Co, Waltham, MA.

Loret, B., Khalili, N., 2000. A three-phase model for unsaturated soils. Int. J. Numer. Anal. Meth. Geomech. 24, 893-927.

Low, P.F., 1987. Structural component of the swelling pressure of clays. Langmuir 3, 18-25.

Ma, C.M., Hueckel, T., 1992. Effects of inter-phase mass transfer in heated clays: a mixture theory. Int. J. Eng. Sci. 30 (11), $1567-1582$.

Mesri, G., Olson, R.E., 1970. Shear-strength of Montmorillonite. Géotechnique 20 (3), 261-270.

Mitchell, J.K., 1993. Fundamentals of Soil Behavior, second ed. Wiley, Chichester.

Murad, M.A., 1999. Thermo-mechanical model for hydration swelling in smectitic clays. Int. J. Num. Anal. Meth. Geomech. 27 (7), Part I: pp. 673-696, Part II: pp. 697-720.

Murray, R.S., Quirk, J.P., 1990. Intrinsic failure and cracking of clay. Soil Sci. Soc. Am. J. 54 (4), 1179-1184.

Norrish, K., 1954. The swelling of montmorillonite. Faraday Soc., London, Discussions 18, 353-359.

Posner, A.M., Quirk, J.P., 1954. Changes in basal spacing of montmorillonite in electrolyte solutions. Colloidal Science 19, $798-812$.

Ransom, B., Helgeson, H.C., 1994. Estimation of the standard molal heat capacities entropies and volumes of 2:1 clay minerals. Geochim. Cosmochim. Acta 58 (2), 4537-4547.

Saiyouri, N., Hicher, P.Y., Tessier, D., 2000. Microstructural approach and transfer water modelisation in highly compacted unsaturated swelling clays. Mech. Frictional Cohesive Mater. 5 (1), 41-66.

Schoen, M., 1996. Computer simulations of the rheological behavior of confined films. Molecular Simulations 17, 369-398.

Sherwood, J.D., 1993. Biot poroelasticity of a chemically active shale. Proc. R. Soc. Lond. 440, 365-377.

Sherwood, J.D., 1994a. Swelling of shale around a cylindrical wellbore. Proc. R. Soc. Lond. 444, 161-184.

Sherwood, J.D., 1994b. A model of hindered solute transport in a poroelastic shale. Proc. R. Soc. Lond. 445, 679-692.

Sposito, G., Prost, R., 1982. Structure of water adsorbed on smectites. Chem. Rev. 82 (6), 552-573.

Sposito, G., Skipper, N.L., Sutton, R., Park, S.-H., Soper, A.K., Greathouse, J.A., 1999. Surface geochemistry of the clay minerals. Proc. Natl. Acad. Sci. USA 96, 3358-3364.

Sridharan, A., Jayadeva, M.S., 1982. Double layer theory and compressibility of clays. Géotechnique 32, $133-144$.

Staverman, A.J., Smit, J.A.M., 1975. Thermodynamics of irreversible processes. Membrane theory. In: van Olphen, H., Mysels, K.J. (Eds.), Physical Chemistry: enriching topics from Colloid and Surface Science. Theorex, La Jolla, CA.

van Olphen, H., 1963. Compaction of clay sediments in the range of molecular particle distances. In: Proceedings of the 11th National Conference on Clays and Clay Minerals. National Academy of Sciences/National Research Council, Washington DC, pp. $178-187$. 